\title{
Study of Model Predictive Control for Path-Following Autonomous Ground Vehicle Control under Crosswind Effect
}

\author{
Fitri Yakub, ${ }^{1}$ Aminudin Abu, ${ }^{1}$ Shamsul Sarip, ${ }^{2}$ and Yasuchika Mori ${ }^{3}$ \\ ${ }^{1}$ Malaysia Japan International Institute of Technology, Universiti Teknologi Malaysia, Jalan Semarak, 54100 Kuala Lumpur, Malaysia \\ ${ }^{2}$ UTM Razak School of Engineering and Advanced Technology, Universiti Teknologi Malaysia, Jalan Semarak, \\ 54100 Kuala Lumpur, Malaysia \\ ${ }^{3}$ Graduate School of System Design, Tokyo Metropolitan University, 6-6 Asahigaoka, Hino, Tokyo 191-0065, Japan
}

Correspondence should be addressed to Fitri Yakub; mfitri.kl@utm.my

Received 4 November 2015; Revised 1 March 2016; Accepted 17 March 2016

Academic Editor: Yongji Wang

Copyright (C) 2016 Fitri Yakub et al. This is an open access article distributed under the Creative Commons Attribution License, which permits unrestricted use, distribution, and reproduction in any medium, provided the original work is properly cited.

\begin{abstract}
We present a comparative study of model predictive control approaches of two-wheel steering, four-wheel steering, and a combination of two-wheel steering with direct yaw moment control manoeuvres for path-following control in autonomous car vehicle dynamics systems. Single-track mode, based on a linearized vehicle and tire model, is used. Based on a given trajectory, we drove the vehicle at low and high forward speeds and on low and high road friction surfaces for a double-lane change scenario in order to follow the desired trajectory as close as possible while rejecting the effects of wind gusts. We compared the controller based on both simple and complex bicycle models without and with the roll vehicle dynamics for different types of model predictive control manoeuvres. The simulation result showed that the model predictive control gave a better performance in terms of robustness for both forward speeds and road surface variation in autonomous path-following control. It also demonstrated that model predictive control is useful to maintain vehicle stability along the desired path and has an ability to eliminate the crosswind effect.
\end{abstract}

\section{Introduction}

Today, model predictive control (MPC) is one of the more popular optimal control techniques which is widely employed for the control of constrained linear or nonlinear systems. MPC uses a mathematical dynamics process model to predict the future behaviour of the system and optimize the process control performance over a prediction horizon [1]. The MPC model can be used easily at different levels of the process control structure, such as multiple input and multiple output dynamics systems that offer attractive solutions for regulation and tracking problems, while guaranteeing stability [2]. Since the end of the 1980s, robust MPCs which explicitly take account of model uncertainties, constraints, and faults in the control actuator, plant-model mismatch, and disturbances or noise have been studied for more practical applications [3]. Numerous research studies have investigated the stability properties of MPC for systems without uncertainty and for uncertain linear systems. Several robust predictive formulations utilize the min-max approach, where the manipulated input trajectory is computed by solving an optimization problem that requires minimizing the objective function and satisfying the input and state constraints over all possible realizations of the uncertainty. These have been applied mainly to impulse response models and state-space approaches by solving a finite horizon open-loop control optimization problem [4]. Due to its advantages, MPC has been implemented in several applications for automotive and other transportation active safety systems, such as active steering, active traction, active braking, and active differentials or suspension systems, in order to coordinate and improve vehicle handling, stability, and ride comfort [5-8].

A vehicle capable of handling many things at once, without any human intervention, can be termed as an autonomous vehicle. Basic functions of autonomous braking and steering however are insufficient; the vehicle has to have the ability to sense its surrounding plus being able to determine desired location, which can be achieved using a variety of instruments and pieces of equipment such as radar, global positioning system, on-board camera for vision, and 
an independent operating unit. All sensory data are then computed for obstacle identification; avoidance is then executed using advanced control system. In this paper, we limit autonomous vehicles to ground vehicles, which are increasingly being studied by several researchers from academia, industry, and military. Several control methods are being used, including fuzzy logic [9], hybrid control [10], H-infinity control [11], and linear quadratic regulators [12]. The best comparative studies on model predictive control strategies for autonomous guidance vehicles can be found in Park et al. [13], Yoon et al. [14], and Falcone et al. [15], where a nonlinear dynamics model of a vehicle is used for the controller design of an active front steering manoeuvre in a double-lane change scenario. Keviczky et al. [16] studied the effect of side wind via an active front steering manoeuvre for an autonomous vehicle using nonlinear MPC. Since nonlinear MPC poses considerable challenges due to its real-time implementation algorithm, many researchers opted for linear MPC for their study.

The assumption of this study are as follows: vehicle trajectory is known, as per Borrelli et al.s [17] study, and the crosswind effect will be assigned as a step response, since the crosswind effect disturbance on the vehicle can be approximated as a constant. In this paper, we extend the concept of MPC for application in vehicle manoeuvring problems where a trajectory optimization is solved at each time step. The trajectory was designed to be a double-lane changing scenario. The autonomous vehicle manoeuvring was simulated in a variety of conditions: low $\left(10 \mathrm{~ms}^{-1}\right)$ and high $\left(30 \mathrm{~ms}^{-1}\right)$ forward speed and low (icy) and high (concrete, wet) road friction surface with the intention of following predetermined trajectory as close as possible while maintaining vehicle stability. The control inputs were front steering angle, rear steering angle, and direct yaw moment control, while the output controls were the yaw angle and lateral vehicle position. Two different controllers were compared to evaluate the performance of the six-degree-of-freedom (6DoF) vehicle model: a simple (2DoF) controller without vehicle roll dynamic and a complex (3DoF) bicycle model controller with roll dynamics. Other performance indexes evaluated were efficacy and robustness of the MPC for the autonomous vehicle in terms of control and stability.

The main objective of this paper is to evaluate the robustness of model predictive control approaches for autonomous path-following car dynamics control with simple and complex models of the vehicle, while rejecting the effects of wind gusts to the system. There are many studies to be consulted in stabilization of the vehicle; examples are two-wheel steering (2WS) $[15,18]$, four-wheel steering (4WS) [19], and 2WS with direct yaw moment control (DYC) $[5,20,21]$ with different control strategies. The first contribution of this paper is the effect of vehicle roll dynamics motion consideration to the system, whereas most previous papers only focused on a $2 \mathrm{DoF}$ vehicle model (lateral and yaw motion). Moreover, based on the authors' knowledge, there is no comparative study for autonomous path-following vehicle control using MPC techniques for three control signal manoeuvres (including the three control signals here); the robustness evaluation discussed here becomes the main novelty of this paper. Furthermore, we would like to investigate the effectiveness of model predictive control manoeuvres in the case of the crosswind effect to the system in a variety of forward speeds and road adhesions. The rest of the paper is organized as follows: Section 2 describes the linear vehicle model, linear tire model, and wind model. Next, a linear model predictive control algorithm concept is explained in Section 3. Section 4 examines and describes the effectiveness of the linear MPC for a car dynamics system on a two-lane change scenario. Lastly, conclusions and future works are given in Section 5.

\section{Vehicle Model}

2.1. Bicycle Model. Figure 1 shows a well-known vehicle model, which is a single-track model based on the simplification that the right and left wheels are lumped in a single wheel at the front and rear axles. The simplified vehicle model used in this paper illustrates the motion movement and dynamics concerning the car vehicle subject to the longitudinal, lateral, yaw, roll, and rotational dynamics of the front and rear wheel motion, represented as $6 \mathrm{DoF}$. The longitudinal, lateral, and yaw dynamics effects are shown in Figure 1(b) as a top view of the car vehicle, and in Figure 1(a), the roll dynamics effect is explained with the nomenclature for a front view of the vehicle. In this paper, the nonlinear vehicle was linearized based on the assumption that $\sin \theta=0$ and $\cos \theta=1$ for both steering angles, the vehicle side slip angle, and the roll angle. We also assumed that the whole vehicle mass is sprung, which is ignoring the suspension and wheel weights for unsprung mass. This linearized model still behaves and represents the actual nonlinear vehicle model at certain operating points of the region. The details of the mathematical calculation for the vehicle model are presented in Chen and Peng [22] for further knowledge.

In this paper, we use the following nomenclature: $F_{x}$ and $F_{y}$ represent the longitudinal and lateral tire forces, respectively, $F_{z}$ is the normal tire load, $F_{w}$ and $M_{w}$ represent the force and moment exerted by the side wind, respectively, $x, y$, and $z$ correspond to the coordinates of the body frame of a car position, $l$ is the vehicle wheelbase, $T_{b}$ is the wheel torque, $v_{x}$ and $v_{y}$ are the longitudinal and lateral wheels velocities, respectively, $\delta_{f}$ and $\delta_{r}$ express the steering angle of the front and rear wheels, respectively, $M_{f}$ and $M_{r}$ represent the reaction yaw moment appearing at the front and rear wheels, respectively, $M_{z}$ is the total reaction of yaw moment of the wheels produced by the DYC, $\mu$ acts as the track friction coefficient, $\psi$ is the yaw/heading angle and $\dot{\psi}$ is the yaw rate, $\beta$ is the vehicle side slip angle, and $\phi$ and $\dot{\phi}$ are the roll and roll rate angles, respectively. The variable at the front and rear wheels is denoted by lower subscripts $(\cdot)_{f}$ and $(\cdot)_{r}$.

The motion of longitudinal, lateral, yaw, roll, and rotational dynamics of the front and rear wheels using a $6 \mathrm{DoF}$ system based on a linear vehicle model is described through the following differential equations [23]:

$$
\begin{aligned}
m \ddot{x}= & m \dot{y} \dot{\psi}+2 F_{x f}+2 F_{x r}-2 h m \dot{\psi} \dot{\phi} \\
m \ddot{y}= & -m \dot{x} \dot{\psi}+2 F_{y f}+2 F_{y r}+h m \ddot{\phi}+F_{w} \\
I_{z z} \ddot{\psi}= & 2 l_{f} F_{y f}-2 l_{r} F_{y f}+I_{x z} \ddot{\phi}+M_{f}+M_{r} \\
& +M_{w}
\end{aligned}
$$




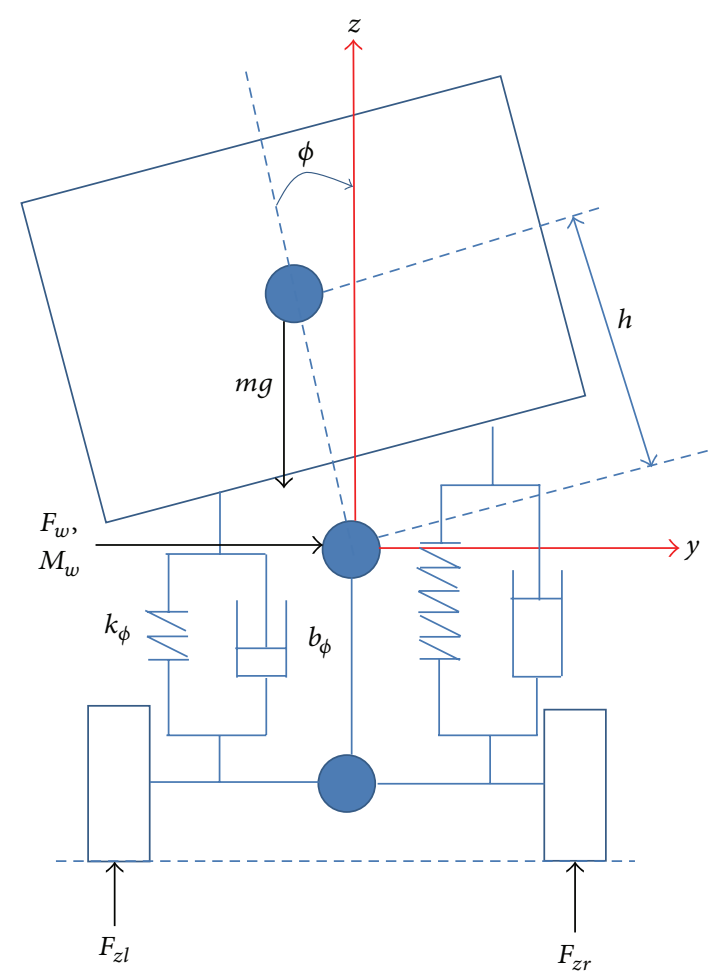

(a) Front view

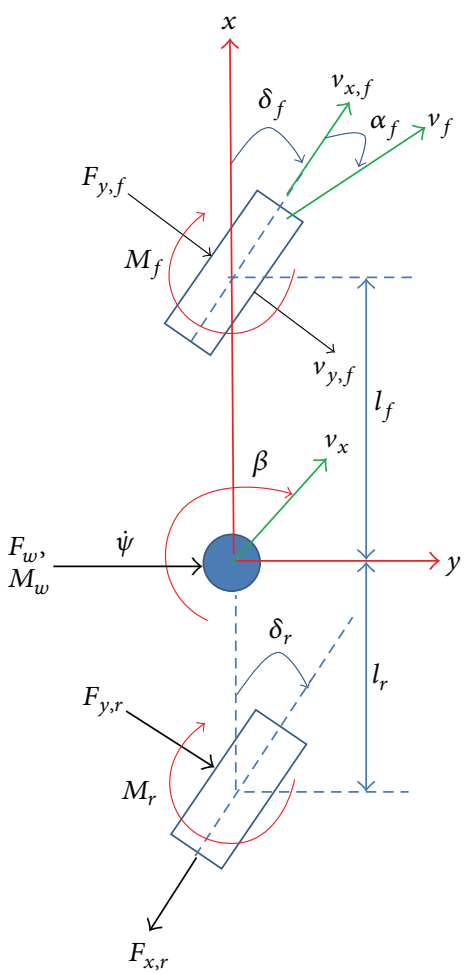

(b) Top view

FIGURE 1: Simplified bicycle model.

$$
\begin{aligned}
\left(I_{x x}+m h^{2}\right) \ddot{\phi}= & m g h \phi-2 k_{\phi} \phi-2 b_{\phi} \dot{\phi} \\
& +m h(\ddot{y}+\dot{\psi} \dot{x})+I_{x z} \ddot{\psi} \\
J_{b} \dot{\omega}_{w i}= & -r_{w} F_{x i}-T_{b i}-b_{w} \omega_{i}, \quad i=(f, r) .
\end{aligned}
$$

The motion equations for the vehicle in an inertial frame or on $y$ - $x$ axis under the assumption of a small yaw angle may be given as

$$
\begin{aligned}
& \dot{X}=\dot{x} \cos \psi-\dot{y} \sin \psi=v_{x}-\dot{y} \psi, \\
& \dot{Y}=\dot{x} \sin \psi+\dot{y} \cos \psi=v_{x} \psi+\dot{y} .
\end{aligned}
$$

2.2. Tire Model. Tire dynamics must be considered for the vehicle model, since the tires are the only contact that the vehicle has with the road surface. Besides the forces of gravity and aerodynamics, all the forces are induced by the tires, which may affect the vehicle chassis, handling, and stability. Their complexity and nonlinear behaviour must also reflect the operating condition of the controller over their whole region throughout varied manoeuvring range in longitudinal, lateral, and roll directions. The most frequently used existing nonlinear tire models of application and structure are determined through key parameters and analytical considerations based on tire measurement data. They are called semiempirical tire models or Pacejka tire model [24].

On the other hand, the nonlinear tire model can also be described linearly for some parts of the operating conditions; therefore in this paper, we will consider the linear

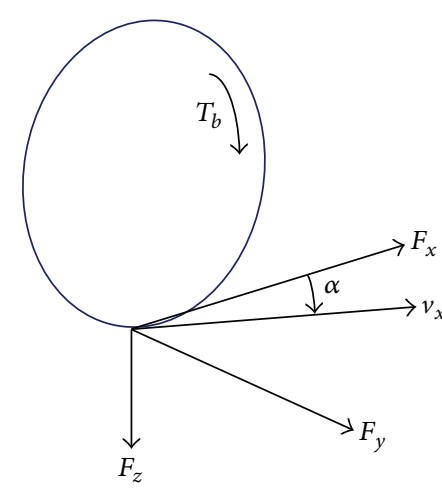

FIgURE 2: Tire model.

tire model. Figure 2 illustrates the terminology used for describing the longitudinal, lateral, and vertical tire forces and their orientation. Thus, the linear tire model is valid under constant normal load forces on the tires, constant longitudinal slip of tires, and neglected aerodynamic drag. The relationship between the longitudinal force, vertical force, and the longitudinal tire slip ratio is given by the following equations:

$$
\begin{aligned}
F_{x} & =\mu_{x}(s) F_{z} \\
s & =\frac{r_{w} \omega_{w}}{v_{x}}-1
\end{aligned}
$$




$$
\begin{aligned}
& F_{z f}=\frac{l_{r} m g}{2 l}, \\
& F_{z r}=\frac{l_{f} m g}{2 l},
\end{aligned}
$$

where $r_{w}$ is the tire's geometric radius, $\omega_{w}$ is the angular velocity of the tires, $v_{x}$ in (3b) is the tire's forward velocity, $F_{x}$ is proportional to the normal force, $F_{z}$, and $\mu_{x}(s)$ represents the longitudinal wheel slip friction coefficient of road adhesions and is a function of slip ratio $s$.

The lateral forces on the front and rear tires are characterized and modelled by a linear function with the front and rear tire slip angles $\alpha_{f}$ and $\alpha_{r}$ denoted by $F_{y, f}$ and $F_{y, r}$, respectively. The linear tire model yields the following expression for the front and rear tire forces:

$$
\begin{gathered}
F_{y, f}=C_{f} \alpha_{f}, \\
F_{y, r}=C_{r} \alpha_{r},
\end{gathered}
$$

where $C_{f}$ and $C_{r}$ are the tire cornering stiffness parameters for the front and rear tires, respectively. The slip angles for the front and rear wheels, with a small angle assumption, are given such that

$$
\begin{gathered}
\alpha_{f}=\frac{v_{y}+l_{f} \dot{\psi}}{v_{x}}-\delta_{f}, \\
\alpha_{r}=\frac{v_{y}-l_{r} \dot{\psi}}{v_{x}}-\delta_{r} .
\end{gathered}
$$

The details of mathematical equations for a linear tire model can be read through in [25]. Assumptions and approximations presented in this paper are representative of the nonlinear tire model at certain regional points; this provides a good balance between capturing the important features and regions of laterally unstable behaviour [26].

2.3. Wind Model. The effect of wind on the stability of the vehicle is an important and primary safety consideration of this paper. A strong gust of wind from the inward or outward side will generate force and torque that could be large enough for a vehicle to roll over or go outside of the lane. The resulting wind pressure forces and torques acting on the rigid body, in general, can be represented by three axes: longitudinal, lateral, and vertical. A general expression of force and torque is given by the following equations:

$$
\begin{aligned}
F_{w} & =\frac{C_{F} \rho A v_{r}^{2}}{2}, \\
M_{w} & =\frac{C_{M} \rho A L v_{r}^{2}}{2} .
\end{aligned}
$$

In the above equations, $A$ is the vehicle area, $L$ is the vehicle length, $L=l_{f}+l_{r}, \rho$ is the density of air, $v_{r}$ is the relative wind speed, and $C_{F}$ and $C_{M}$ are the force and moment nondimensional coefficients, respectively. The wind speed or crosswind is represented by $v_{w}$ as shown in Figure 3. In

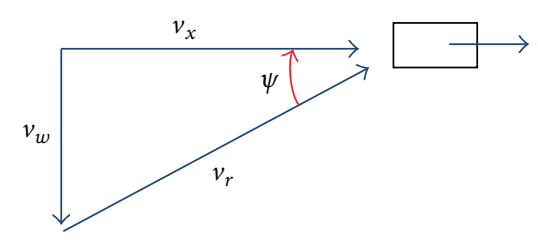

FIgURE 3: Vehicle wind speed in crosswind situation.

general, crosswind can be at various angles, but, for simplicity, in this paper we will assume the crosswind to be at a 90degree angle and will focus on the wind's impact on the lateral forces and yaw torques [27].

The vehicle motion in ((1a), (1b), (1c), (1d), (1e))-(6) can be described by the following compact differential equation:

$$
\begin{aligned}
& \dot{x}=A x+B_{1} u+B_{2} w+B_{3} r, \\
& y=C x+D u
\end{aligned}
$$

with $x \in R^{x}, u \in R^{u}, w \in R^{w}, r \in R^{r}$, and $y \in R^{y}$ representing the state vectors, control input vectors, crosswind effects as a disturbance vector, desired trajectory vectors, and measured output vectors, respectively. We define

$$
\begin{aligned}
& x=\left[\begin{array}{llllllll}
v_{y} & Y & \dot{\psi} & \psi & \dot{\phi} & \phi & \omega_{f} & \omega_{r}
\end{array}\right]^{T}, \\
& u=\left[\begin{array}{lll}
\delta_{f} & \delta_{r} & M_{z}
\end{array}\right]^{T} \text {, } \\
& w=\left[\begin{array}{ll}
F_{w} & M_{w}
\end{array}\right]^{T}, \\
& r=\left[\begin{array}{ll}
Y_{\mathrm{des}} & \psi_{\mathrm{des}}
\end{array}\right]^{T} \text {, } \\
& h(\xi)=\left[\begin{array}{ll}
Y & \psi
\end{array}\right]^{T} \text {. }
\end{aligned}
$$

The state vectors represent the states for lateral velocity, lateral position, yaw rate, yaw angle, roll rate, roll angle, and front and rear wheels angular speed, respectively, and the output vectors represent lateral position and yaw angle.

\section{Linear Model Predictive Control}

MPC has been used as a highly effective and very successful control scheme with the simple design framework proving to be a practical controller able to combine multivariable systems and utilize online optimization process control. The stability guarantees of existing predictive control approaches for nonlinear systems with uncertainty, however, remain contingent upon the assumption of the initial feasibility of the optimization, and the set of initial conditions, starting from where feasibility of the optimization problem and, therefore, stability of the closed-loop system is guaranteed, is not explicitly characterized [28]. For example, the occurrence of faults in control actuators adds another layer of complexity to the problem of controller design for nonlinear uncertain systems. Note that the stability guarantees provided by a controller may no longer hold in the presence of faults in the control actuators that prevent the implementation of the control action prescribed by the control law, and these 


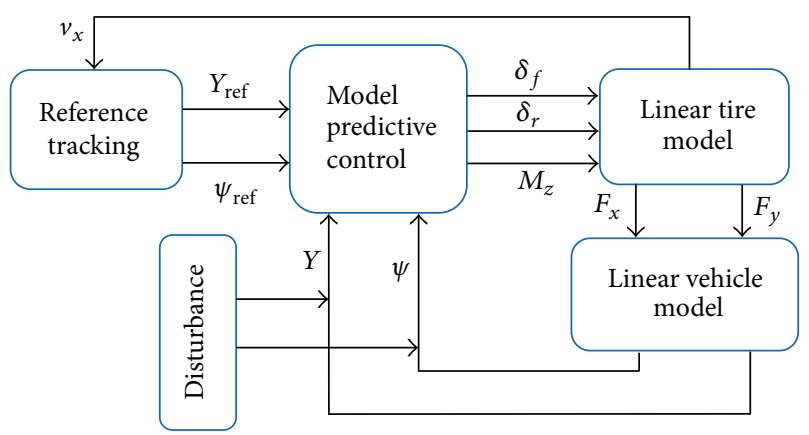

Figure 4: Predictive control structure.

faults can have substantial negative ramifications owing to the interconnected nature of processes [29].

In order to implement MPC with a receding horizon control strategy, the following strategy method is adopted:

(1) A dynamics process model is used to predict the behaviour of the plant and future plant outputs at each instant $k$ based on both previous and the latest observations of the system's inputs and outputs.

(2) The control signal inputs are calculated by minimizing the error of tracking between the predicted output and desired trajectory signal to keep the process as close as possible to following the trajectory, while considering the objective functions and constraints.

(3) Only the first control signal is implemented on the plant, whilst others are rejected in anticipation of the next sampling instant, where the future output will be known.

(4) Step (1) is repeated with updated values and all orders are updated.

In this paper, we use the linear model of predictive control. The hierarchical control structure is adopted in MPC, as shown in Figure 4. Figure 4 illustrates the control structure for $2 \mathrm{WS}$ that uses front steering only, $\delta_{f}$, for $4 \mathrm{WS}$ that uses front and rear steering, $\delta_{f}$ and $\delta_{r}$, and for 2WS with DYC that produces external yaw moment $\left(M_{z}\right)$ at both front and rear wheels as a control input to the system. These systems are used to control the vehicle in order to follow a given reference trajectory. They include the vehicle speed, desired reference trajectory, modelled predictive control, and linear vehicle model with linear tire model.

Using the equations from the vehicle and tire model, as explained and defined in (7), the basic equations of linear vehicle motion can be given as follows:

$$
\begin{aligned}
m \dot{v}_{y} & =\frac{1}{I_{x x} v_{x}}\left[-\left(\mu C_{r}+C_{f}\right) J_{x q} v_{y}\right. \\
& \left.+\left(\mu\left(C_{r} l_{r}-C_{f} l_{f}\right) J_{x q}-I_{x x} m v_{x}^{2}\right) \dot{\psi}\right] \\
& -\frac{1}{I_{x x}}\left[\left(h b_{\phi}\right) \dot{\phi}-h\left(m g h-k_{\phi}\right) \phi-\mu C_{f} J_{x q} \delta_{f}\right. \\
& \left.+\mu C_{r} J_{x q} \delta_{r}\right]
\end{aligned}
$$

$$
\begin{aligned}
& I_{z z} \ddot{\psi}=\frac{1}{v_{x}}\left[\mu\left(C_{r} l_{r}-C_{f} l_{f}\right) v_{y}\right. \\
& \left.\quad-\mu\left(C_{f} l_{f}^{2}+C_{r} l_{r}^{2}\right) \dot{\psi}\right]+\mu C_{f} l_{f} \delta_{f}-\mu C_{r} l_{r} \delta_{r} \\
& \quad+M_{z} \\
& I_{x x} \ddot{\phi}=\frac{h}{v_{x}}\left[\mu\left(C_{r} l_{r}-C_{f} l_{f}\right) \dot{\psi}-\mu\left(C_{f}+C_{r}\right) v_{y}\right] \\
& \quad-b_{\phi} \dot{\phi}+\left(m g h-k_{\phi}\right) \phi+\mu C_{f} h \delta_{f}+\mu C_{r} h \delta_{r},
\end{aligned}
$$

where $J_{x q}=I_{x x}+m h^{2}$. A DYC that produces the reaction moment occurring at the front and rear wheels due to the steering angle effect (as an external yaw moments $\left(M_{z}\right)$ ) can be approximated with the following equations:

$$
\begin{gathered}
M_{f} \approx 2 l_{f} C_{f} M_{z}, \\
M_{r} \approx 2 l_{r} C_{r} M_{z} .
\end{gathered}
$$

We define front steering angle, rear steering angle, and direct yaw moment control as the inputs to the system. Thus, the vehicle motion can be represented in a given discrete statespace structure, as follows:

$$
\begin{aligned}
x_{l}(k+1 \mid k)= & A_{l} x_{l}(k \mid k)+B_{l} u_{l}(k \mid k) \\
& +B_{r} r_{l}(k \mid k), \\
y_{l}(k \mid k)= & C_{l} x_{l}(k \mid k)+D_{l} u_{l}(k \mid k),
\end{aligned}
$$

where $x_{l}(k \mid k)$ is the state vector at time step $k$ and $x_{l}(k+$ $1 \mid k)$ is the state vector at time step $k+1$, with $x_{l}(k \mid k) \epsilon$ $R^{x_{l}(k \mid k)}, u_{l}(k \mid k) \in R^{u_{l}(k \mid k)}, r_{l}(k \mid k) \in R^{r_{l}(k \mid k)}$, and $y_{l}(k \mid k) \epsilon$ $R^{y_{l}(k \mid k)}$ representing the state vectors, control input vectors, reference vectors, and measured output vectors, respectively. We define

$$
\begin{aligned}
& x_{l}(k)=\left[\begin{array}{llllll}
v_{y} & Y & \dot{\psi} & \psi & \dot{\phi} & \phi
\end{array}\right]^{T} \text {, } \\
& r_{l}(k)=\left[\begin{array}{ll}
Y_{\mathrm{des}} & \psi_{\mathrm{des}}
\end{array}\right]^{T}, \\
& y_{l}(k)=\left[\begin{array}{ll}
Y & \psi
\end{array}\right]^{T} \text {. }
\end{aligned}
$$

For 2WS, 4WS, and 2WS with DYC, the control signal to the systems with the same tuning control parameters is given as follows:

$$
\begin{aligned}
& u_{l}(k)=\left[\begin{array}{l}
\delta_{f}
\end{array}\right], \\
& u_{l}(k)=\left[\begin{array}{ll}
\delta_{f} & \delta_{r}
\end{array}\right]^{T}, \\
& u_{l}(k)=\left[\begin{array}{ll}
\delta_{f} & M_{z}
\end{array}\right]^{T} .
\end{aligned}
$$

We formulate the optimization of the predictive control system, which takes the constraints imposed on the input and input rate, respectively, as given in the following form:

$$
\begin{aligned}
-u_{l} & \leq u_{l}(k) \leq+u_{l}, \\
-\Delta u_{l} & \leq \Delta u_{l}(k) \leq+\Delta u_{l} .
\end{aligned}
$$


One approach to maintain closed-loop stability would be to use all available control actuators (13) so that even if one of the control actuators fails, the rest can maintain closed-loop stability which the reliable control approaches. The use of redundant control actuators, however, incurs possibly preventable operation and maintenance costs. These economic considerations dictate the use of only as many control loops as is required at a time. To achieve tolerance with respect to faults, control-loop reconfiguration can be carried out in the event of failure of the primary control configuration based on the assumption of a linear system.

Based on the linear vehicle model in (11) and by defining the outputs in (12), we consider the following cost functions to the system:

$$
\begin{aligned}
J\left(x_{l}(k), U_{l k}\right)= & \sum_{i=1}^{H_{p}}\left\|\tilde{y}_{l}(k+i \mid k)-r(k+i \mid k)\right\|_{Q_{i}}^{2} \\
& +\sum_{i=0}^{H_{c}-1}\left\|\Delta \widetilde{u}_{l}(k+i \mid k)\right\|_{R_{i}}^{2}
\end{aligned}
$$

In (15), the first summation of the given cost function reflects the reduction of trajectory tracking errors among the predicted outputs $\tilde{y}_{l}(k+i \mid k)\left(i=0, \ldots, H_{p}-1\right)$ and the output reference signals $r(k+i \mid k)\left(i=0, \ldots, H_{p}-1\right)$, and the second summation reflects on the penalization of the control signal effort $\Delta \widetilde{u}_{l}(k+i \mid k)\left(i=0, \ldots, H_{c}-1\right)$ of the steering control manoeuvre. The aim of the predictive control system is to determine the optimal control input vector $\Delta \tilde{u}_{l}(k+i \mid k)$ so that the error function between the reference signal and the predicted output is reduced.

The difference in the steering angle $\Delta \tilde{u}_{l}(k+i \mid k)$ is given in the case that the cost function is at a minimum value. The weight matrices $Q(i)$ and $R(i)$ are semipositive definite and positive definite, respectively, and can be tuned for any desired closed-loop performance. $Q(i)$ is defined as the state tracking weight, since the error $\tilde{y}_{l}(k+i \mid k)-r(k+i \mid k)$ can become as small as possible by increasing $Q(i)$. In a similar fashion, $R(i)$ represents the input tracking weight and the variation of the input is decreased to slow down the response of the system by increasing $R(i)$. The predictive and control horizon are typically considered to be $H_{p} \geq H_{c}$, while the control signal is considered constant for $H_{c} \leq i \leq H_{p}$. Matrices $Q(i)$ and $R(i)$ represent the matrix weight of their appropriate dimensions for outputs and inputs, respectively, giving the state feedback control law as follows:

$$
u_{l}\left(k, y_{l}(k)\right)=u_{l}(k-1)+\Delta u_{l}\left(y_{l}(k)\right) .
$$

An optimal input was computed for the following time step rather than being calculated for the immediate time step by addressing convex optimization problems during each time step. After that, the first input is applied on the plant in (11), and the others are rejected, which yield the optimal control sequence as (16). The optimization problem in (15) is iterated with the new value at a time $k+1$ via new state $x(k+1)$, and all orders are then updated.

The required direct yaw moment control $M_{z}$ is obtained from the variation between the two sides of the vehicle torque, as in (1e). In this research, the braking torque is only used based on the yaw rate; in other words, it is only activated in the case that the vehicle moves toward instability or in emergency manoeuvres, since it impacts the longitudinal motion, while the rear steering angle is assumed for the total manoeuvre to be in control or in standard driving manoeuvres. We take into account three control inputs, that is, front and rear steering angles, as well as differential braking at rear right and left tire, but only one control input is used at a time [30].

\section{Simulation Results}

4.1. Scenario Description. The linear model predictive controller explained and presented was implemented for a vehicle path following a double-lane change scenario with a crosswind effect present throughout the simulation. A double-lane change manoeuvre approximates as an emergency manoeuvre case and generally demonstrates the agility and capabilities of the vehicle in lateral and roll dynamics. During such a manoeuvre, an understeering or oversteering, or even a rollover situation, may occur. In this scenario, the vehicle is considered travelling horizontally or straightforward, following the path with a constant velocity of $10 \mathrm{~ms}^{-1}$ and $30 \mathrm{~ms}^{-1}$ without braking or accelerating. The drag force and torque given by (6) in the initial driving conditions are assumed to act in the direction of the path at time $t=1 \mathrm{sec}$ with a wind velocity of $v_{w}=10 \mathrm{~ms}^{-1}$. The forces and torques arising from this sidewise acting wind gust are assumed to be persistent and are applied as step functions throughout the simulation time, as shown in Figure 5. Table 1 shows the sport utility passenger car model parameters and their definitions used in this paper, based on Solmaz et al. [31].

For stability analysis, we simulated the system with only one input-in particular, the front steering angle $\delta_{f}$ or $2 \mathrm{WS}$. We set the vehicle velocity to be constant at $v_{x}=15 \mathrm{~ms}^{-1}$; the road adhesion coefficient $\mu=1$ and wind velocity $v_{w}=$ $10 \mathrm{~ms}^{-1}$. Figure 6(a) shows a stability analysis based on the root locus of the open-loop system in (7) showing matrix $A$ without the crosswind effect $\left(B_{2}=0\right)$ and references signal $\left(B_{3}=0\right)$. Those settings contribute to a stable region for both outputs (vehicle side slip and yaw rate responses). Matrix $A$ is given in (8) with input signal being front steering angle $\left(B_{1}=\delta_{f}\right)$. Since the root locus command in MATLAB is only working on the single-input single-output, therefore we evaluate separately the output response with single input signal.

Figure 6(b) illustrates the effect of these factors: a crosswind effect $\left(B_{2} \neq 0\right)$, references signal $\left(B_{3}=0\right)$, and control signal $\left(B_{1}=0\right)$, with the same matrix $A$ as in (8) that yields unstable responses for both vehicle side slip and yaw rate. We can also notice that the poles are different for both cases due to $B_{2}$ matrices that are based on the crosswind effect. Furthermore, Figure 6(b) shows that within a wind velocity, $v_{w}=10 \mathrm{~ms}^{-1}$, the system is stable with the appropriate matrices of $A, B_{1}$, and $B_{2}$. Figure 6(a) also illustrates that paired $A$ and $B_{1}$ are controllable, based on the model parameters listed in Table 1.

In this paper, the predictive controller was implemented by minimizing vehicle deviation from the target path to 

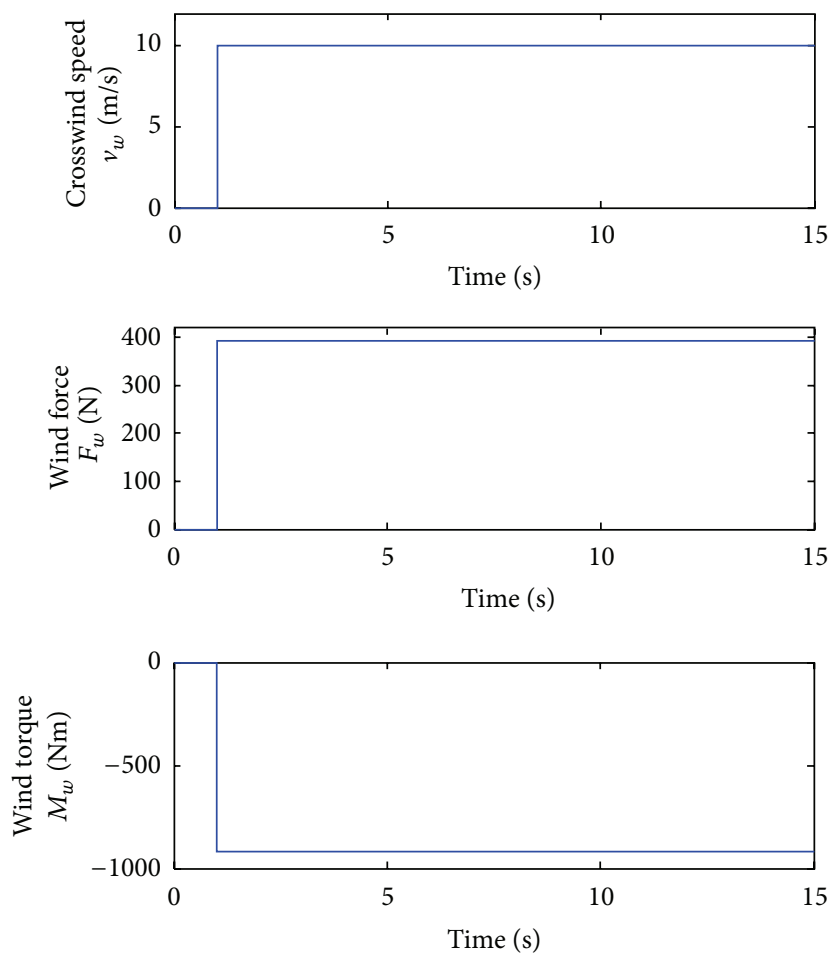

FIGURE 5: Force and torque for crosswind speed of $10 \mathrm{~ms}^{-1}$.

TABLE 1: Car vehicle parameters.

\begin{tabular}{lc}
\hline Parameter & Value \\
\hline Car mass $[\mathrm{kg}]$ & 1300 \\
Inertia around the roll, $x$-axis $\left[\mathrm{kgm}^{2}\right]$ & 370 \\
Inertia around $z$-axis $\left[\mathrm{kgm}^{2}\right]$ & 2167.56 \\
Distance of front and rear wheels from centre of gravity $(\mathrm{CoG})[\mathrm{m}]$ & $1.45,1.07$ \\
Distance between the vehicle CoG and the assumed roll axis $[\mathrm{m}]$ & 0.45 \\
Equivalent suspension roll damping coefficient $[\mathrm{Nms}]$ & 4200 \\
Equivalent suspension roll stiffness coefficient $[\mathrm{Nm}]$ & 40000 \\
Linear approximation of tire stiffness for front and rear tire $[\mathrm{N} / \mathrm{rad}]$ & 133000,121000 \\
Gravitational constant $\left[\mathrm{m} / \mathrm{s}^{2}\right]$ & 9.8 \\
\hline
\end{tabular}

achieve the main aim, which is to follow the desired or reference trajectory as close as possible. The controllers are compared against each other for $2 \mathrm{DoF}$ and $3 \mathrm{DoF}$ bicycle models, which excludes vehicle roll dynamics at various speeds, specifically low $\left(10 \mathrm{~ms}^{-1}\right)$ and high $\left(30 \mathrm{~ms}^{-1}\right)$, and also under wet concrete $(\mu=0.7)$ and icy $(\mu=0.1)$ road surfaces.

Since our study focused on an autonomous vehicle, it is reasonable to assume that the controllers will adapt to the road or environment, such as in the case of road adhesions, adverse weather, visual cues, traffic, and overall relevant driving conditions. Therefore, both controllers (2DoF and $3 \mathrm{DoF}$ ) were designed and implemented with the parameters and conditions given in Tables 2, 3, and 4 for different vehicle forward speeds and road adhesion coefficients. The pathfollowing tracking error is a measurement of how closely the output responses follow the reference trajectory, which is a measure of the deviation from the benchmark. In this paper, we use the standard deviation of the root-mean square formula to calculate the tracking error, given by the following equation:

$$
e_{t}=\sqrt{\operatorname{Var}\left(y_{o}-y_{r}\right)}=\sqrt{\frac{1}{(n-1)} \sum\left(y_{o}-y_{r}\right)^{2}},
$$

where $e_{t}$ is the tracking error, $n$ represents the number of time periods, and $y_{o}$ and $y_{r}$ express the measured output and reference.

4.2. Results and Discussions. Prior to proposed simulations, we carried out standard vehicle manoeuvres tests to validate our model in the open-loop simulation scenario. Standard tests available are the J-turn, fishhook, and double-lane change [32], which are representative of on-road manoeuvres without tripping (caused by external force from road curb or 

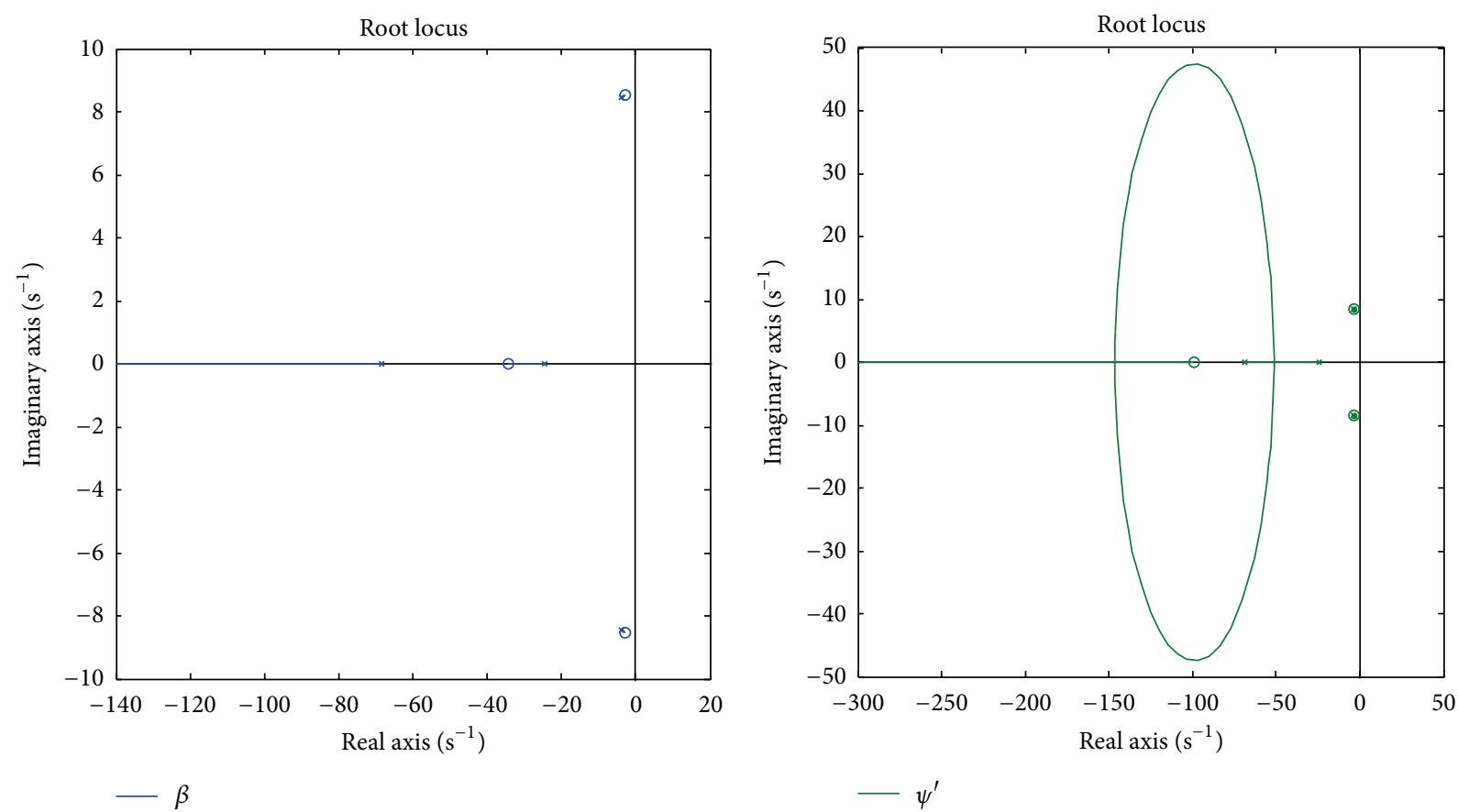

(a) Without crosswind effect
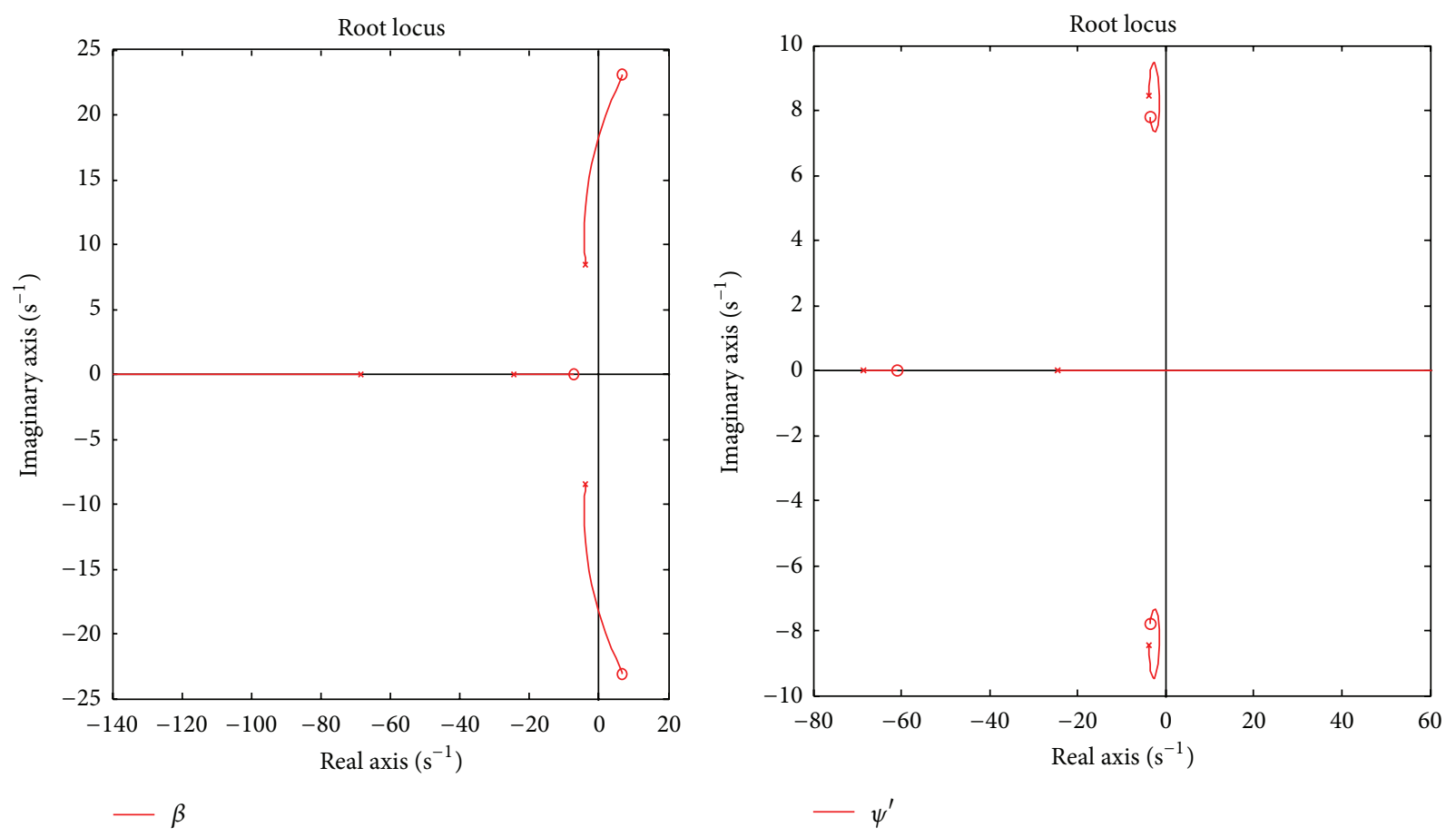

(b) With crosswind effect

Figure 6: Open-loop system in (7).

collision with other vehicles). We performed only the doublelane change and roll rate feedback fishhook test, for vehicle validation purposes in the open-loop simulation, as shown in Figures 7 and 8 . The vehicle speed was set at $30 \mathrm{~ms}^{-1}$, which is suitable for both tests, with a road surface coefficient of 0.1 . As illustrated in Figures 7 and 8, the vehicle response in terms of yaw rate, roll rate, and lateral acceleration has been proven to be validated, thus being suitable for other simulations.

For easier comparison between controllers' performances, in achieving vehicle stabilization when subjected to various conditions, controllers tuning parameters from Tables 3 and 4 were selected as suitable. The weighting gains 

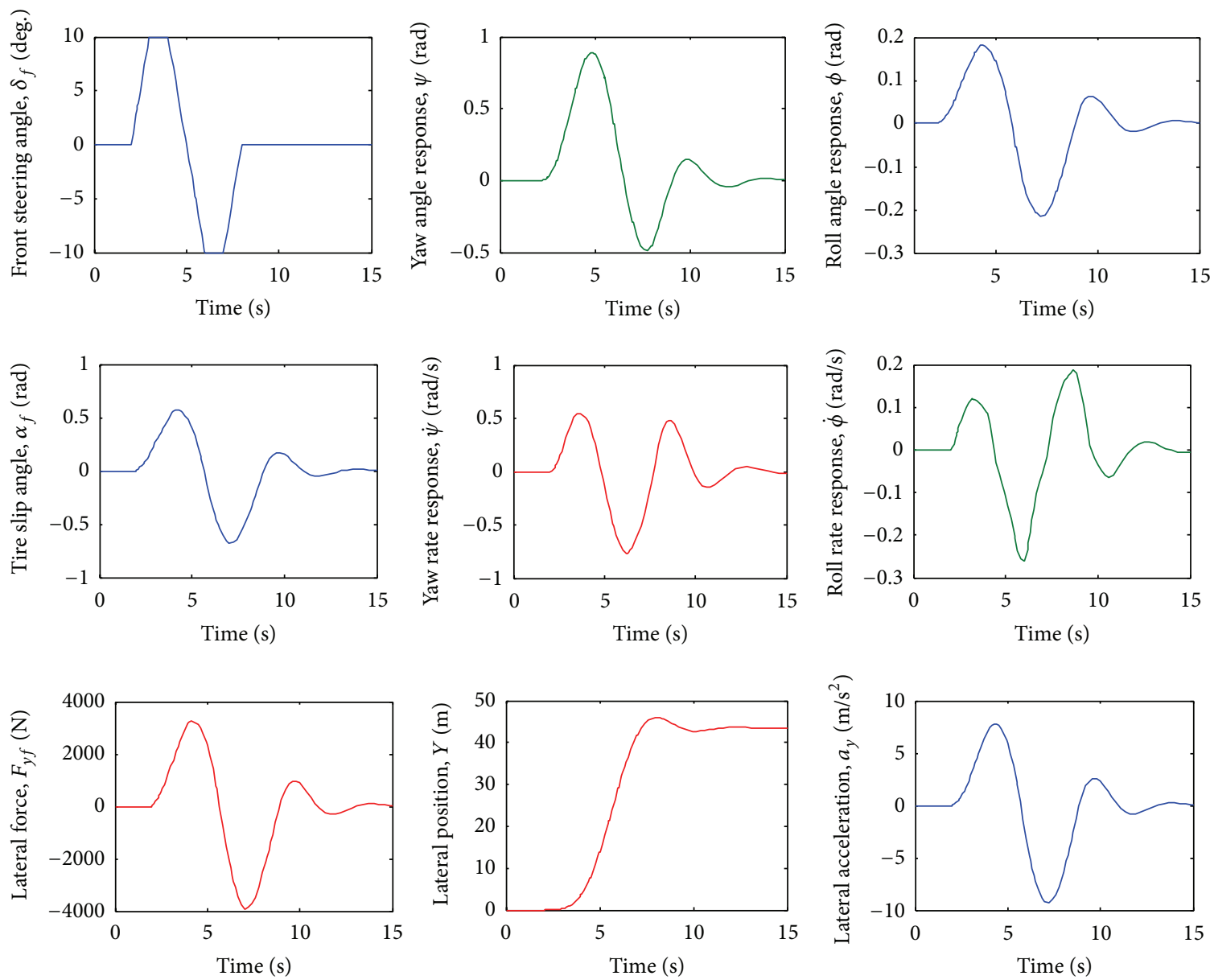

FIGURE 7: Vehicle manoeuvre test of single-lane change at $30 \mathrm{~ms}^{-1}$ with $\mu=0.1$.

TABLE 2: Model predictive control parameters.

\section{Parameter}

Predictive horizon

Control horizon

Sampling time $[\mathrm{s}]$

Constraints on max and min steering angles [deg.]

Constraints on max and min changes in steering angles [deg./s]

Constraints on max and min changes in moment torque [Nm/rad]

for controllers input and output were selected using trialand-error method, where selection criteria were based on the best response output for both input and output for weighting gains.

The first simulation revolves around various road surface friction coefficients, from wet concrete $(\mu=0.7)$ to icy surface condition $(\mu=0.1)$, with a constant forward speed of $10 \mathrm{~ms}^{-1}$. The MPC weighting tuning parameters are listed in Table 3. We evaluated the controller's robustness for the output responses by comparing the performance of $2 \mathrm{WS}$, $4 \mathrm{WS}$, and 2WS with DYC manoeuvres at a forward speed of
$10 \mathrm{~ms}^{-1}$, as shown in Figures 9-11. The controller tracking errors, based on (17) for lateral position and yaw angle responses, are summarized in Table 5. From Figure 9, there are not many differences between both controllers under the conditions of lowest speed and high road adhesion coefficient; both controllers yielded perfect response when following a given trajectory, while maintaining vehicle stability and rejecting external crosswind effect. It can be seen that, for 4WS and 2WS with DYC manoeuvres, the rear steering angle and the direct yaw moment are almost unused, since the front steering can provide sufficient control ability. This 

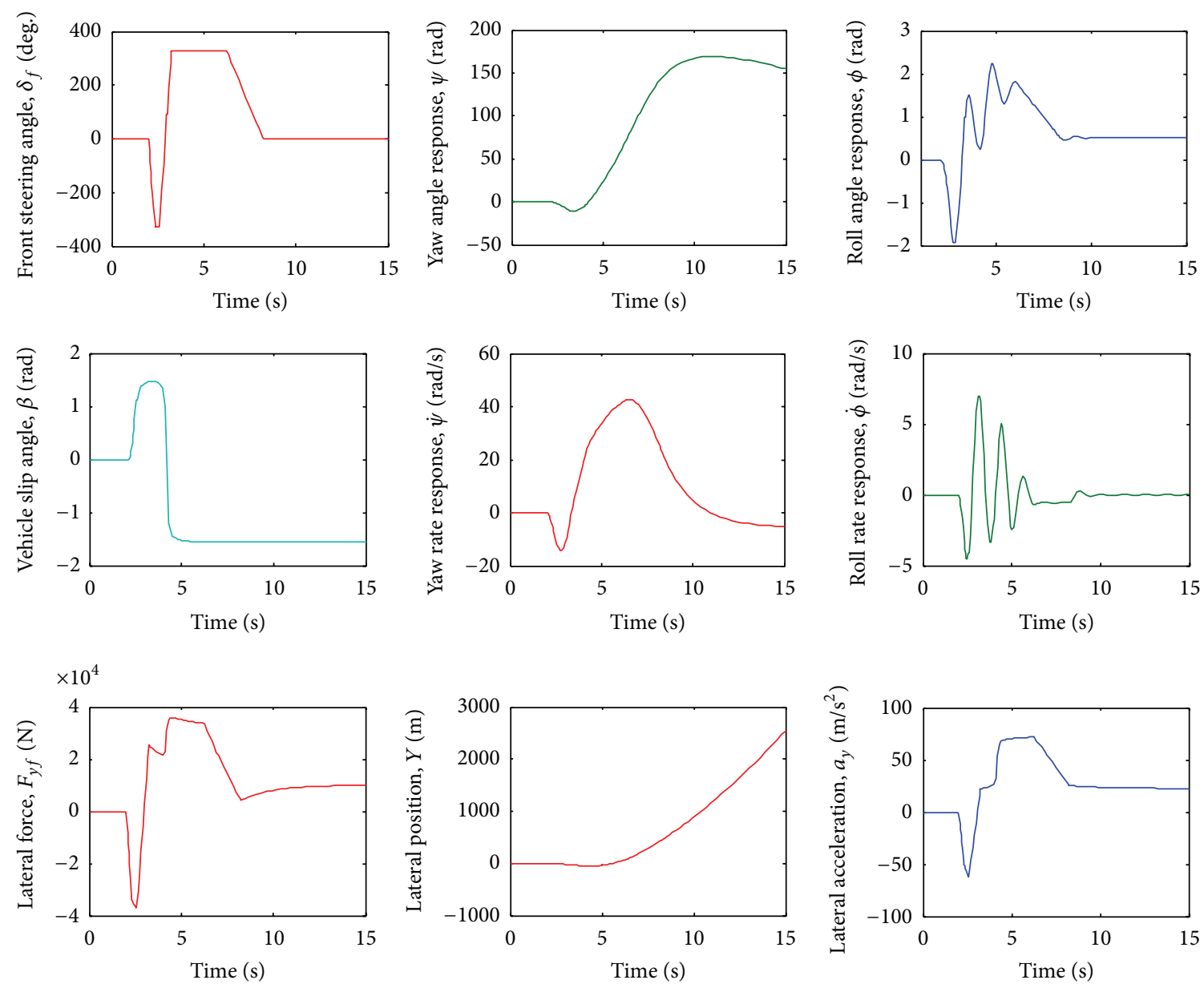

FIGURE 8: Vehicle manoeuvre test of roll rate feedback at $30 \mathrm{~ms}^{-1}$ with $\mu=0.1$.

TABLE 3: Model predictive control weighting matrices parameters for $v_{x}=10 \mathrm{~ms}^{-1}$.

\begin{tabular}{|c|c|c|c|}
\hline & uvres & $2 \mathrm{DoF}$ & $3 \mathrm{DoF}$ \\
\hline \multirow{4}{*}{$2 \mathrm{WS}$} & \multirow{2}{*}{$v_{x}=10 \mathrm{~ms}^{-1}, \mu=0.7$} & $R_{1}=0.1, \Delta R_{1}=0.03$ & $R_{1}=0.1, \Delta R_{1}=0.03$ \\
\hline & & $Q_{11}=1.95, Q_{22}=0.5$ & $Q_{11}=5.25, Q_{22}=0.5$ \\
\hline & \multirow{2}{*}{$v_{x}=10 \mathrm{~ms}^{-1}, \mu=0.1$} & $R_{1}=0.1, \Delta R_{1}=0.03$ & $R_{1}=0.1, \Delta R_{1}=0.03$ \\
\hline & & $Q_{11}=1.52, Q_{22}=0.5$ & $Q_{11}=3.95, Q_{22}=0.5$ \\
\hline \multirow{6}{*}{$4 \mathrm{WS}$} & \multirow{3}{*}{$v_{x}=10 \mathrm{~ms}^{-1}, \mu=0.7$} & $R_{1}=0.1, \Delta R_{1}=0.03$ & $R_{1}=0.1, \Delta R_{1}=0.03$ \\
\hline & & $R_{2}=0.1, \Delta R_{2}=0.03$ & $R_{2}=0.1, \Delta R_{2}=0.03$ \\
\hline & & $Q_{11}=1.95, Q_{22}=0.5$ & $Q_{11}=4.5, Q_{22}=0.5$ \\
\hline & \multirow{3}{*}{$v_{x}=10 \mathrm{~ms}^{-1}, \mu=0.1$} & $R_{1}=0.1, \Delta R_{1}=0.03$ & $R_{1}=0.1, \Delta R_{1}=0.03$ \\
\hline & & $R_{2}=0.1, \Delta R_{2}=0.03$ & $R_{2}=0.1, \Delta R_{2}=0.03$ \\
\hline & & $Q_{11}=1.53, Q_{22}=0.5$ & $Q_{11}=3.5, Q_{22}=0.5$ \\
\hline \multirow{6}{*}{$2 \mathrm{WS}+\mathrm{DYC}$} & \multirow{3}{*}{$v_{x}=10 \mathrm{~ms}^{-1}, \mu=0.7$} & $R_{1}=0.1, \Delta R_{1}=0.03$ & $R_{1}=0.1, \Delta R_{1}=0.03$ \\
\hline & & $R_{2}=0.1, \Delta R_{2}=0.03$ & $R_{2}=0.1, \Delta R_{2}=0.03$ \\
\hline & & $Q_{11}=1.93, Q_{22}=0.5$ & $Q_{11}=3.7, Q_{22}=0.5$ \\
\hline & \multirow{3}{*}{$v_{x}=10 \mathrm{~ms}^{-1}, \mu=0.1$} & $R_{1}=0.1, \Delta R_{1}=0.03$ & $R_{1}=0.1, \Delta R_{1}=0.03$ \\
\hline & & $R_{2}=0.1, \Delta R_{2}=0.03$ & $R_{2}=0.1, \Delta R_{2}=0.03$ \\
\hline & & $Q_{11}=1.52, Q_{22}=0.5$ & $Q_{11}=3.5, Q_{22}=0.5$ \\
\hline
\end{tabular}



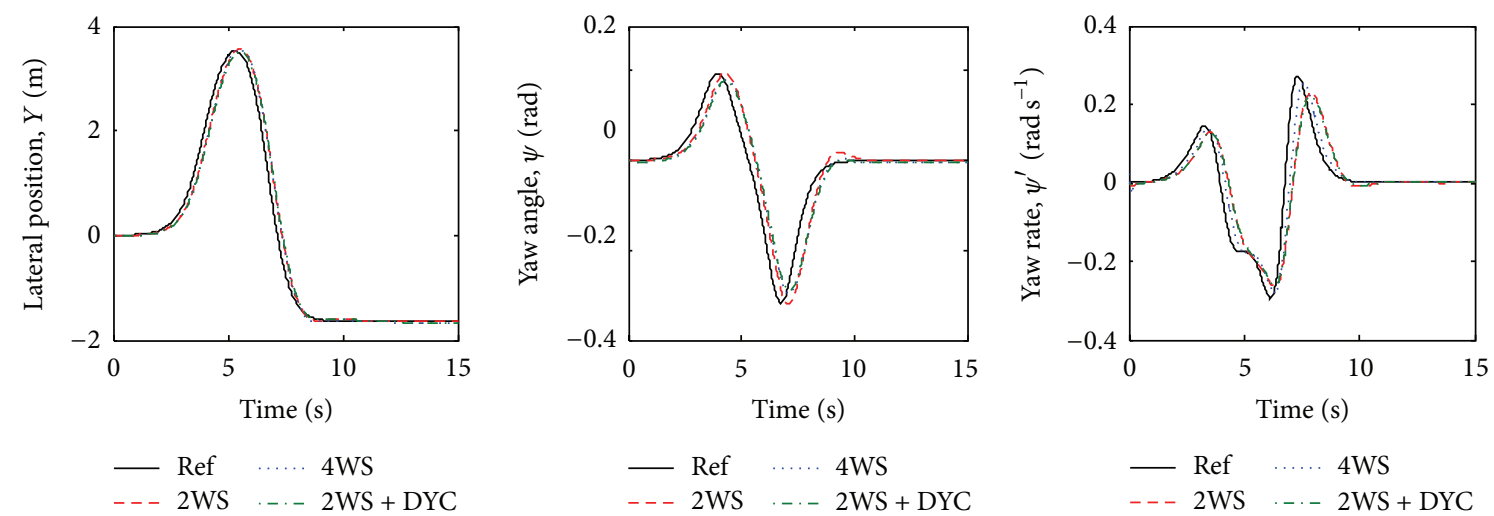

$---2 W S \quad-.2$ WS + DYC
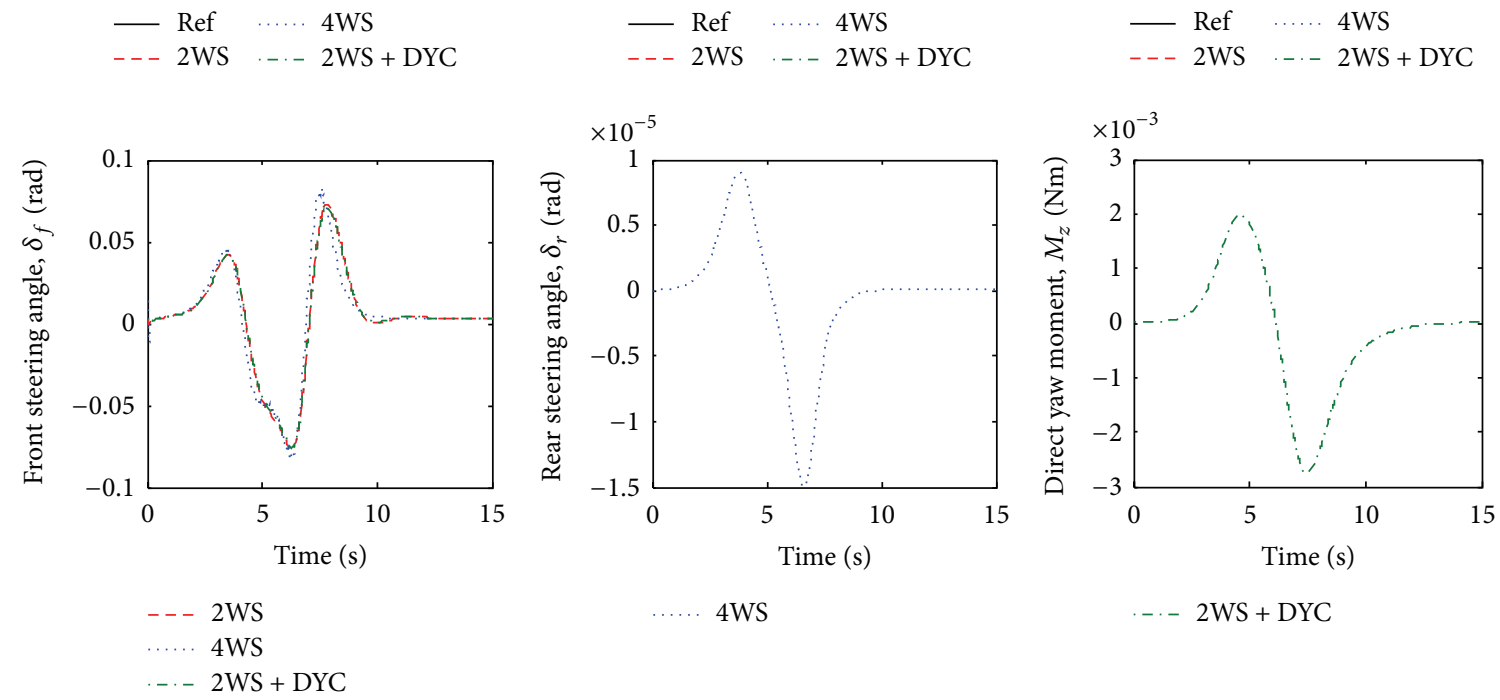

FIGURE 9: 2DoF controller at $10 \mathrm{~ms}^{-1}$ and $\mu=0.7$ by $2 \mathrm{WS}, 4 \mathrm{WS}$, and $2 \mathrm{WS}+\mathrm{DYC}$.

may be partly due to the fact that the rear steering and DYC were not used in lower speed manoeuvres.

Moreover, when the road surface friction was set to icy, the output responses from both controllers and all control manoeuvre techniques did not perfectly follow the desired trajectory as shown in Figures 10 and 11. 2WS with DYC yielded the best performance output compared to the others, especially in yaw rate response. $4 \mathrm{WS}$ was the next best, following 2WS, where the lateral position and yaw angle look almost identical. It seems that the rear steering and direct yaw moment controls were used in conjunction with front steering for vehicle stabilization along the trajectory. However, from Table 5, the controller with $3 \mathrm{DoF}$ gives slightly better tracking error performance manoeuvres compared to controller 2DoF due to the fact that roll dynamics will not have much influence during low speed manoeuvres. Another fact is that, at low vehicle speed, all control inputs (front steering angle, rear steering angle, and direct yaw moment control) are under constraints.

Next, we tested the vehicle at various road friction coefficients again; however this time the vehicle was tested under a high forward speed of $30 \mathrm{~ms}^{-1}$. The same MPC design was used, of which the parameter controls are listed in Table 4. We compared the simulation results for $2 \mathrm{WS}, 4 \mathrm{WS}$, and $2 \mathrm{WS}$ with DYC control manoeuvres for both controllers and present the comparison in Figures 12-15. The simulation results show that, for $2 \mathrm{DoF}$ controller at $30 \mathrm{~ms}^{-1}$ and on wet concrete $(\mu=0.7)$, all control manoeuvres give slightly similar outputs performances. Better responses were achieved in lateral positioning, and although the tracking performance of yaw angle and yaw rate deteriorated, the system still allowed the vehicle to track and follow the trajectory, successfully rejecting the effects of wind gust that impact the vehicle.

As tabulated in Table 6, 4WS and 2WS with DYC show slightly better tracking performance compared to 2WS only. However, in the case of the $3 \mathrm{DoF}$ controller, it can be clearly seen that 2WS with DYC and 4WS control manoeuvres offered a much higher performing response, especially in yaw angle and yaw rate response, than 2WS. This is shown in Figure 13. In this scenario, the rear steering and direct yaw moment control was fully utilized to control and enhance vehicle stability. It is therefore very important to consider roll dynamics in order to enhance vehicle stability and to follow the path trajectory. From Figures 12 and 13, there are some strong frequency oscillations in front of some responses; that is, front lateral force, vehicle side slip angle, and lateral acceleration trace, based on authors' knowledge, come from the numerical calculation, or associated glitches, and initial condition of the system.

Furthermore, when we simulated vehicle manoeuvre for high speed $\left(30 \mathrm{~ms}^{-1}\right)$ and icy road condition $(\mu=0.1)$ for $2 \mathrm{DoF}$ controller, it can be seen that all manoeuvres control 

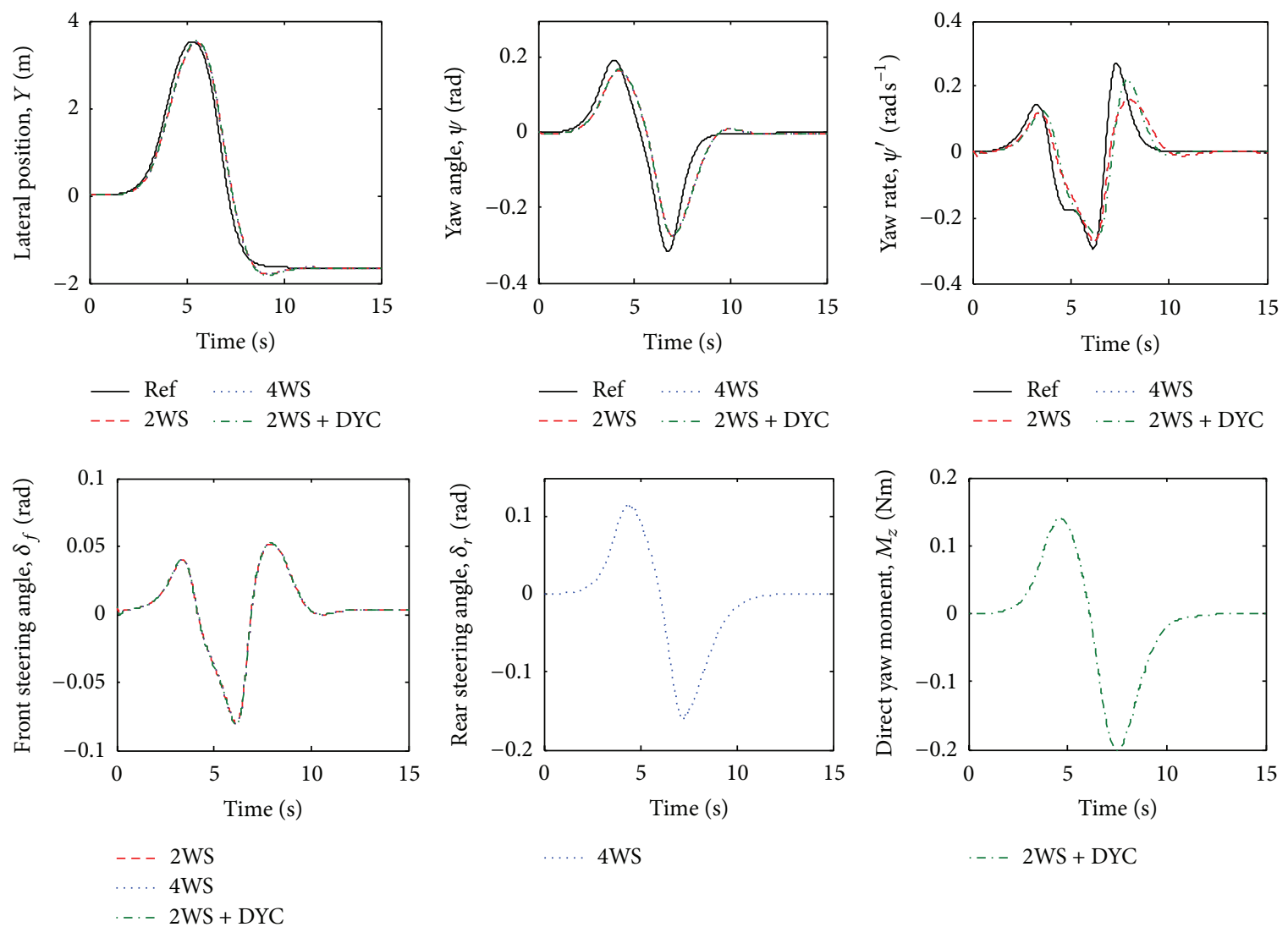

FIgURe 10: 2DoF controller at $10 \mathrm{~ms}^{-1}$ and $\mu=0.1$ by $2 \mathrm{WS}, 4 \mathrm{WS}$, and $2 \mathrm{WS}+\mathrm{DYC}$.

TABLE 4: Model predictive control weighting matrices parameters for $v_{x}=30 \mathrm{~ms}^{-1}$.

\begin{tabular}{|c|c|c|c|}
\hline \multicolumn{2}{|c|}{ Control manoeuvres } & $2 \mathrm{DoF}$ & 3DoF \\
\hline 2WS & $\begin{array}{l}v_{x}=30 \mathrm{~ms}^{-1}, \mu=0.7 \\
v_{x}=30 \mathrm{~ms}^{-1}, \mu=0.1\end{array}$ & $\begin{array}{c}R_{1}=0.1, \Delta R_{1}=0.03 \\
Q_{11}=5.65, Q_{22}=0.5 \\
R_{1}=0.1, \Delta R_{1}=0.03 \\
Q_{11}=0.083, Q_{22}=0.042\end{array}$ & $\begin{array}{c}R_{1}=0.1, \Delta R_{1}=0.03 \\
Q_{11}=3.55, Q_{22}=0.5 \\
R_{1}=0.1, \Delta R_{1}=0.03 \\
Q_{11}=0.03, Q_{22}=0.45\end{array}$ \\
\hline $4 \mathrm{WS}$ & $\begin{array}{l}v_{x}=30 \mathrm{~ms}^{-1}, \mu=0.7 \\
v_{x}=30 \mathrm{~ms}^{-1}, \mu=0.1\end{array}$ & $\begin{array}{c}R_{1}=0.1, \Delta R_{1}=0.03 \\
R_{2}=0.1, \Delta R_{2}=0.03 \\
Q_{11}=1.55, Q_{22}=2.5 \\
R_{1}=0.1, \Delta R_{1}=0.03 \\
R_{2}=0.1, \Delta R_{2}=0.03 \\
Q_{11}=0.005, Q_{22}=0.001\end{array}$ & $\begin{array}{l}R_{1}=0.1, \Delta R_{1}=0.03 \\
R_{2}=0.1, \Delta R_{2}=0.03 \\
Q_{11}=1.35, Q_{22}=3.5 \\
R_{1}=0.1, \Delta R_{1}=0.03 \\
R_{2}=0.1, \Delta R_{2}=0.03 \\
Q_{11}=0.04, Q_{22}=0.5\end{array}$ \\
\hline $2 \mathrm{WS}+\mathrm{DYC}$ & $v_{x}=30 \mathrm{~ms}^{-1}, \mu=0.7$ & $\begin{array}{c}R_{1}=0.1, \Delta R_{1}=0.03 \\
R_{2}=0.1, \Delta R_{2}=0.03 \\
Q_{11}=5.05, Q_{22}=0.5 \\
R_{1}=0.1, \Delta R_{1}=0.03 \\
R_{2}=0.1, \Delta R_{2}=0.03 \\
Q_{11}=0.092, Q_{22}=0.04\end{array}$ & $\begin{array}{l}R_{1}=0.1, \Delta R_{1}=0.03 \\
R_{2}=0.1, \Delta R_{2}=0.03 \\
Q_{11}=3.5, Q_{22}=0.5 \\
R_{1}=0.1, \Delta R_{1}=0.03 \\
R_{2}=0.1, \Delta R_{2}=0.03 \\
Q_{11}=0.5, Q_{22}=2.5\end{array}$ \\
\hline
\end{tabular}

simulation; the tracking responses become unstable and impossible to control, especially under the crosswind effect as shown in Figure 14. The most probable cause is the roll dynamic motion neglect in the $2 \mathrm{DoF}$ controller design, when the vehicle itself was modelled by including roll dynamic factor. It can be said that the inclusion of roll dynamic factor is important for vehicle manoeuvre, in terms of stability and controllability, at high speed and icy road condition. Since high speed coupled with icy road condition will render the equation to become highly nonlinear, it is impossible for a linear tire model to react positively, since the handling properties may be significantly different from those generated 

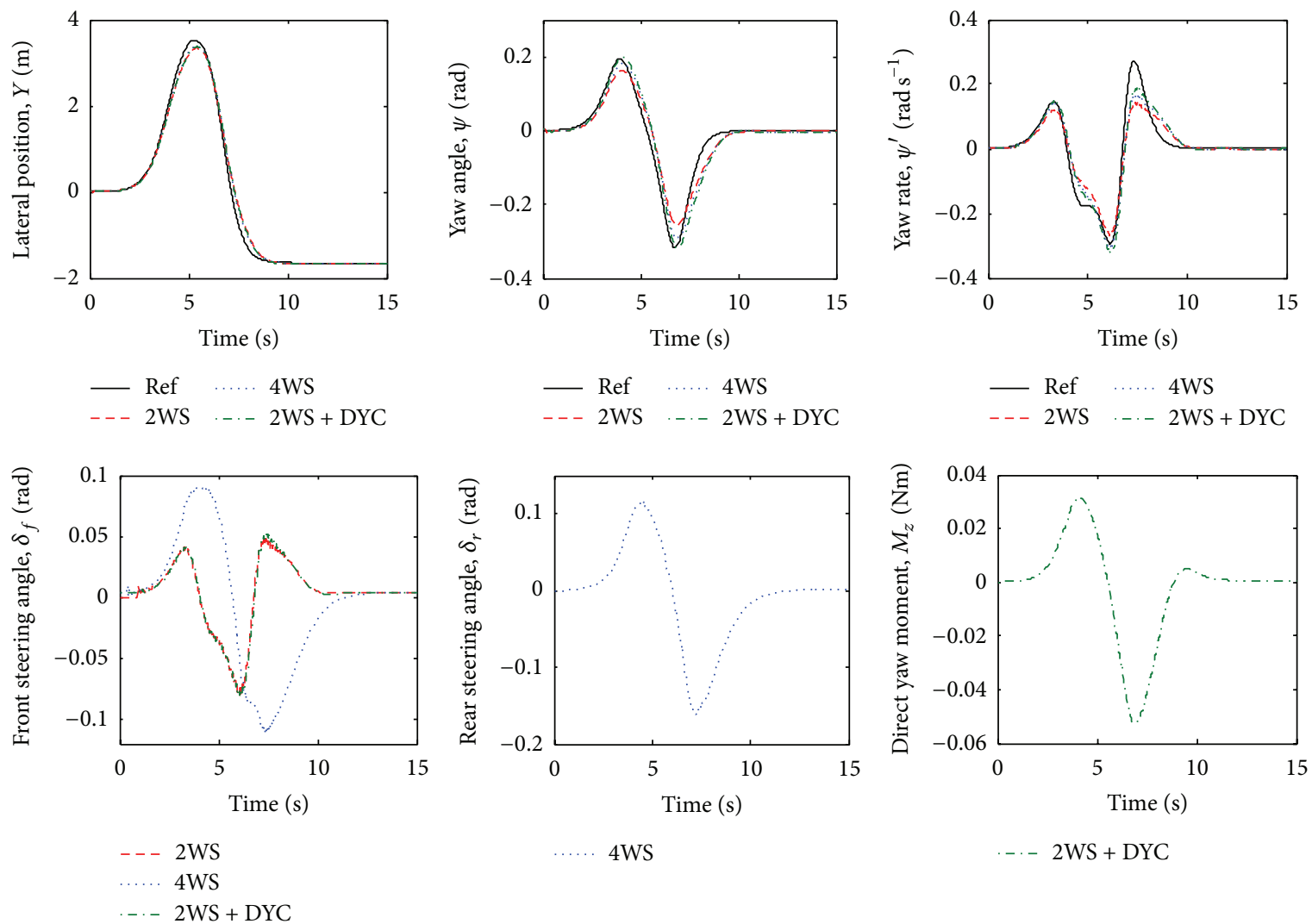

FIGURE 11: 3 DoF controller at $10 \mathrm{~ms}^{-1}$ and $\mu=0.1$ by $2 \mathrm{WS}, 4 \mathrm{WS}$, and $2 \mathrm{WS}+\mathrm{DYC}$.

TABLE 5: Path following tracking errors with road friction surface $\mu=0.7$.

\begin{tabular}{|c|c|c|c|c|c|}
\hline \multirow{2}{*}{ Vehicle speed } & \multirow{2}{*}{ Manoeuvre control } & \multicolumn{2}{|c|}{ Controller 2DoF } & \multicolumn{2}{|c|}{ Controller 3DoF } \\
\hline & & $Y[\mathrm{~m}]$ & $\Psi[\mathrm{rad}]$ & $Y[\mathrm{~m}]$ & $\Psi[\mathrm{rad}]$ \\
\hline \multirow{3}{*}{10} & $2 \mathrm{WS}$ & 0.0637 & 0.0085 & 0.0623 & 0.0074 \\
\hline & 4WS & 0.0548 & 0.0054 & 0.0526 & 0.0051 \\
\hline & $2 \mathrm{WS}+\mathrm{DYC}$ & 0.0542 & 0.0051 & 0.0527 & 0.0051 \\
\hline \multirow{3}{*}{30} & $2 \mathrm{WS}$ & 0.0639 & 0.5348 & 0.0616 & 0.5215 \\
\hline & 4WS & 0.0558 & 0.5239 & 0.0528 & 0.0023 \\
\hline & $2 \mathrm{WS}+\mathrm{DYC}$ & 0.0549 & 0.5226 & 0.0524 & 0.0254 \\
\hline
\end{tabular}

by the linear tire model. These results show that linear tire model is only suitable for analyzing a stable vehicle behaviour under the assumption of small steering and acceleration.

Next, for the 3DoF controller simulation, 2WS with DYC manoeuvres provides a much better tracking response in comparison to $4 \mathrm{WS}$, particularly in the case of lateral output. On the contrary, 4WS manoeuvre demonstrates a much better tracking response in the yaw angle and yaw rate responses, as tabulated in Table 6. With appropriate weight tuning gain, the rear steering angle and direct yaw moment control are fully optimized in order to become stable along the given trajectory. However, for the 2WS manoeuvre, the vehicle responses become unstable, despite several instances where the weighting tuning gains are adjusted for output and input gains. With the inclusion of another input control to the controller design, we can enhance the vehicle responses for both
2WS with DYC and 4WS control manoeuvres show. This means that, for 2WS controller design, it may have to use more than just front steering to stabilize the vehicle under the effects of wind gusts. Figure 15 shows that rear steering angle and direct yaw moment are fully utilized in order to stabilize the vehicle for 2WS with DYC and 4WS manoeuvres control.

In contrast to the $2 \mathrm{DoF}$ controller as shown in Figure 14, in the $3 \mathrm{DoF}$ controllers, the 2WS control manoeuvre can still be used to track a given trajectory, despite not perfectly following the trajectory in a satisfactory manner, especially under crosswind effects. In Figure 15, we can see some oscillations in few traces responses, at the end of the responses. Based on the authors' knowledge, these oscillations may come from the vehicle model and some initial conditions of the system with imperfect controllers tuning weighting gains. In these conditions, neither 2WS nor 4WS control manoeuvres 

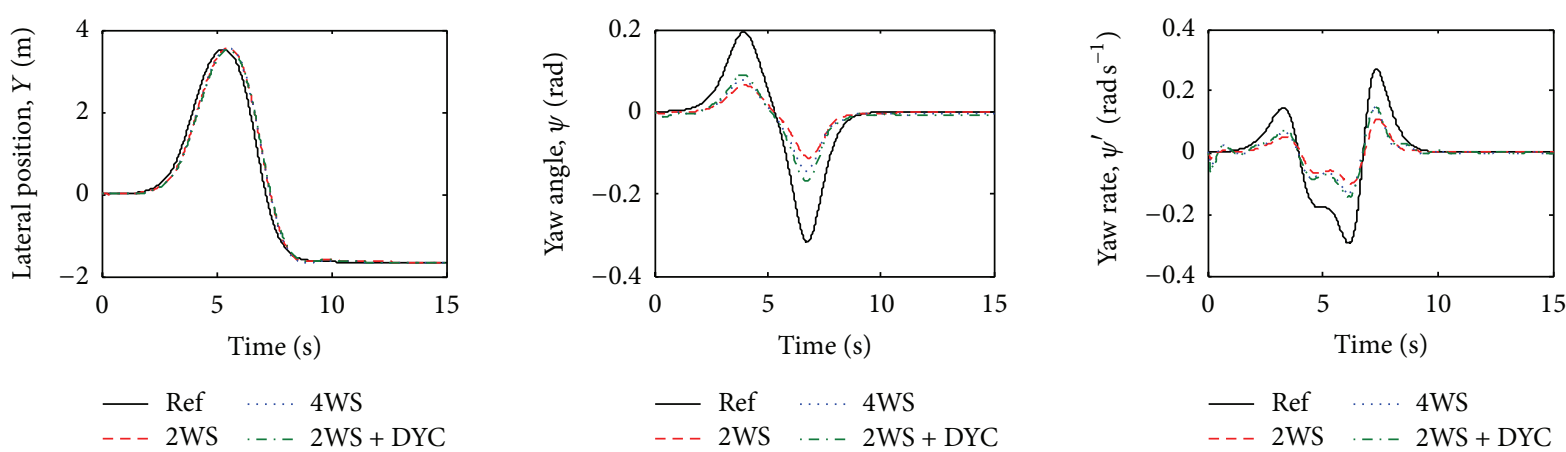

-- $2 \mathrm{WS}$
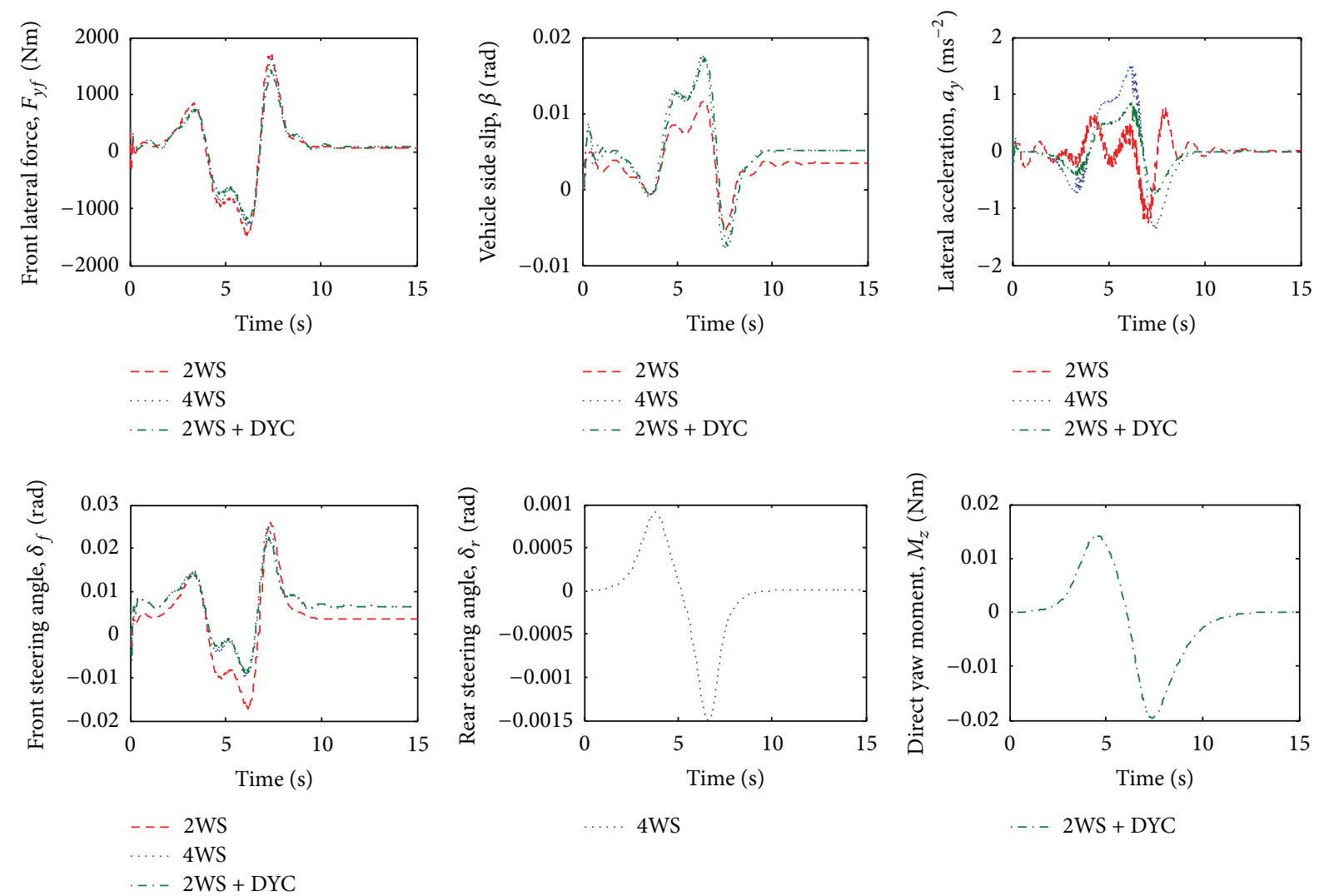

FIGURE $12: 2 \mathrm{DoF}$ controller at $30 \mathrm{~ms}^{-1}$ and $\mu=0.7$ by $2 \mathrm{WS}, 4 \mathrm{WS}$, and $2 \mathrm{WS}+\mathrm{DYC}$.

for lateral response and neither 2WS nor 2WS with DYC control manoeuvres for yaw angle or yaw rate response performed well, causing an increase in vehicle instability, increased vibration, and tracking responses deterioration. We will next focus on how to enhance the controller in order to stabilize vehicle manoeuvrability and handling stability.

We can therefore conclude that, for $4 \mathrm{WS}$, the rear wheels were helping the car to steer by improving the vehicle handling at high speed, while decreasing the turning radius at low speed, as shown by the control signal in Figures 12 and 13. Meanwhile, for 2WS with DYC, active front steering was used in low speed manoeuvres for lateral acceleration, while inclusion of DYC was adopted for high lateral acceleration when the tires were saturated and could not produce enough lateral force for vehicle control and stability as intended. In 2 WS vehicles, the rear set of wheels do not play an active role in controlling the steering. From Table 5 it can be seen that, among the three controllers, 4WS gave the best performance by reducing the tracking error for lateral and yaw angle responses when compared with 2WS with DYC and 2WS only. Tables 5 and 6 tabulated the MPC robustness and the tracking errors in (17) for all types of manoeuvre at $10 \mathrm{~ms}^{-1}$ and $30 \mathrm{~ms}^{-1}$ vehicle speeds and under various road surfaces. Furthermore, all control manoeuvre signals for both controllers were within the input constraints. We would like to highlight that, in MPC approaches, although there was an advantage in multivariable systems, in this study there was a trade-off between the weighting tuning parameter to focus on either lateral position or yaw angle trajectory. In this paper, we have chosen lateral position as the weighting tuning priority, so we may get a better response on yaw angle and yaw rate response by sacrificing lateral position precision. 

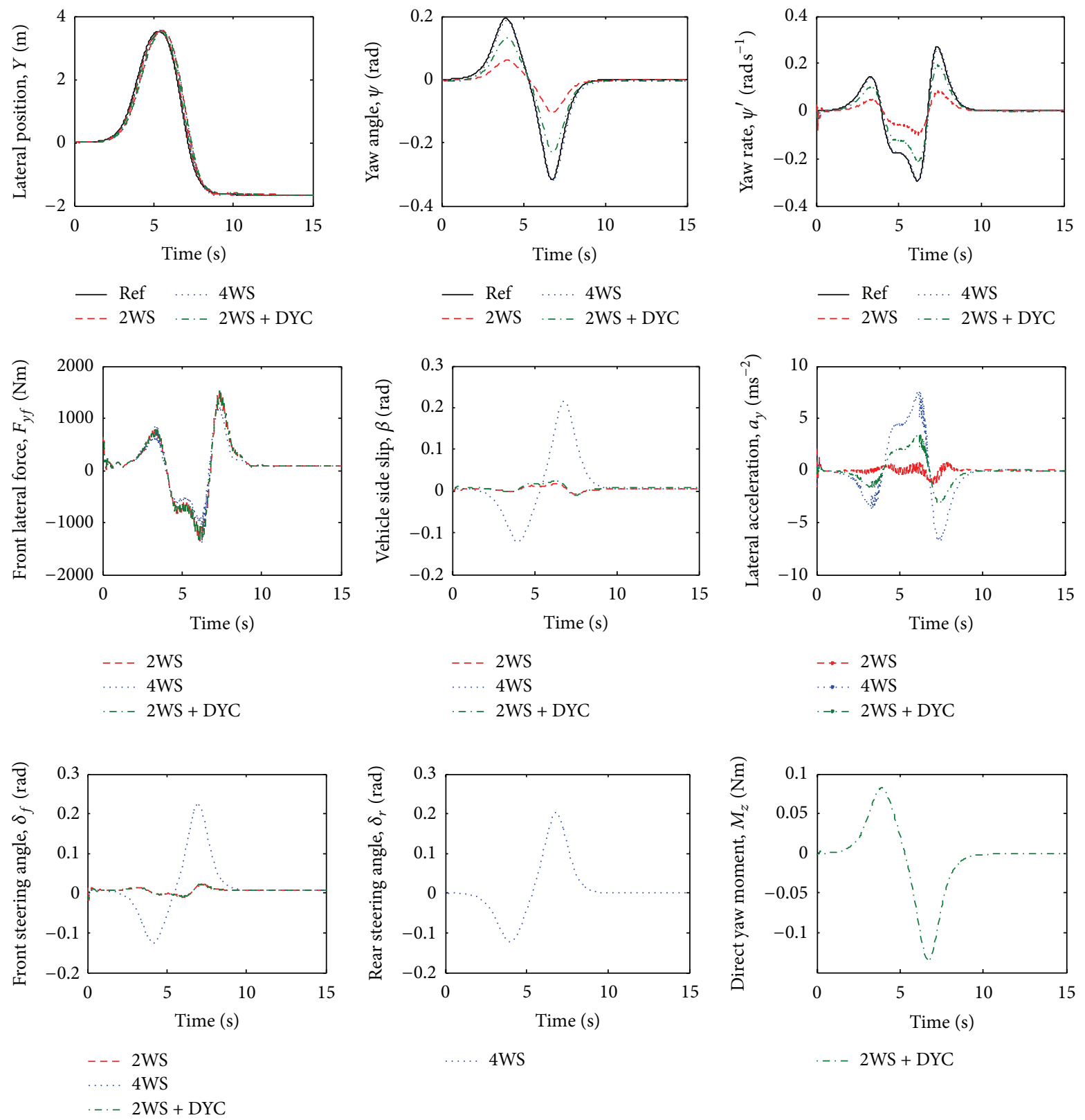

FIGURE 13: 3DoF controller at $30 \mathrm{~ms}^{-1}$ and $\mu=0.7$ by $2 \mathrm{WS}, 4 \mathrm{WS}$, and $2 \mathrm{WS}+\mathrm{DYC}$.

TABLE 6: Path following tracking errors with road friction surface $\mu=0.1$.

\begin{tabular}{|c|c|c|c|c|c|}
\hline \multirow{2}{*}{ Vehicle speed } & \multirow{2}{*}{ Manoeuvre control } & \multicolumn{2}{|c|}{ Controller 2DoF } & \multicolumn{2}{|c|}{ Controller 3DoF } \\
\hline & & $Y[\mathrm{~m}]$ & $\Psi[\mathrm{rad}]$ & $Y[\mathrm{~m}]$ & $\Psi[\mathrm{rad}]$ \\
\hline \multirow{3}{*}{10} & $2 \mathrm{WS}$ & 0.0874 & 0.0115 & 0.0841 & 0.0104 \\
\hline & 4WS & 0.0838 & 0.0109 & 0.0832 & 0.0094 \\
\hline & $2 \mathrm{WS}+\mathrm{DYC}$ & 0.0838 & 0.0110 & 0.0835 & 0.0083 \\
\hline \multirow{3}{*}{30} & $2 \mathrm{WS}$ & Uncontrolled & Uncontrolled & 2.1352 & 0.5264 \\
\hline & 4WS & Uncontrolled & Uncontrolled & 0.8824 & 0.2982 \\
\hline & $2 \mathrm{WS}+\mathrm{DYC}$ & Uncontrolled & Uncontrolled & 0.9964 & 0.4580 \\
\hline
\end{tabular}



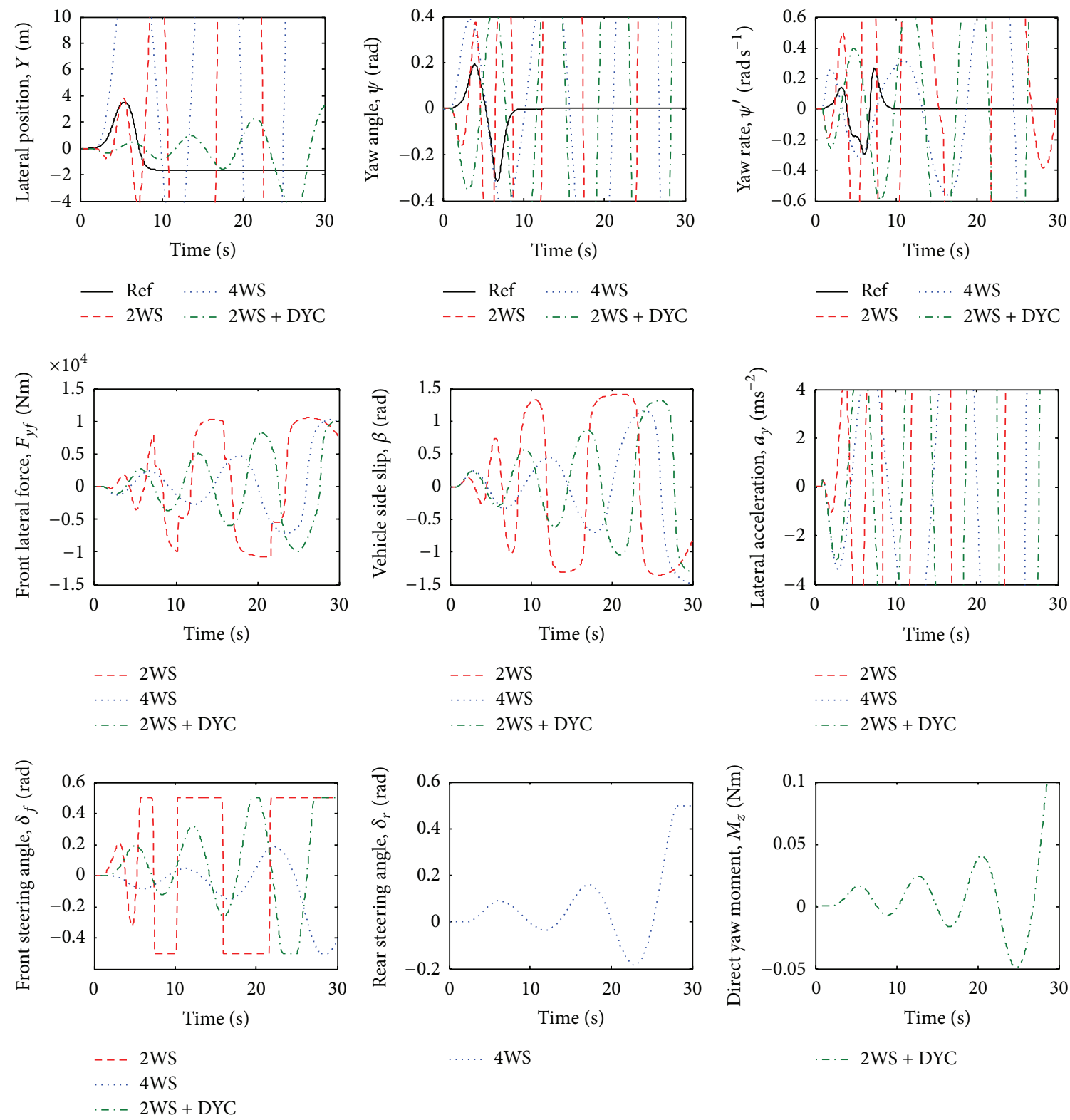

FIGURE 14: $2 \mathrm{DoF}$ controller at $30 \mathrm{~ms}^{-1}$ and $\mu=0.1$ by $2 \mathrm{WS}, 4 \mathrm{WS}$, and $2 \mathrm{WS}+\mathrm{DYC}$.

\section{Conclusion}

This paper presents the robustness of model predictive control for an autonomous ground vehicle on a path-following control, with consideration of wind gust effects subjected to the variation of forward speeds and road surfaces condition, in a double-lane change scenario. The predictive controllers were designed based on two- (lateral-yaw model) and three- (lateral-roll model) degree-of-freedom vehicle models. The only difference between the models is the roll dynamics factor, which is taken into consideration, and both controllers were implemented into a six-degree-of-freedom vehicle model. Based on linear vehicle and tire model approximations of a known trajectory, we evaluated the effect and impact of roll dynamics at low and high forward vehicle speeds and at various road frictions (wet concrete to icy roads) in order to follow a desired trajectory as close as possible while rejecting the crosswind effects and maintaining vehicle stability. We also evaluated and compared the efficiency of the different manoeuvres via two-wheel steering, four-wheel steering, and two-wheel steering with direct yaw moment control.

The simulation results showed that, with the inclusion of roll dynamics in the linear vehicle model, the vehicle stability and adherence to trajectory was considerably improved for the case of high speed manoeuvres on a higher road surface friction coefficient $(\mu=0.7)$ and improved even more for the case of a lower road surface coefficient $(\mu=0.1)$. Furthermore, the obtained results showed and proved that the rear wheels and direct yaw moment are beneficial to help steering 

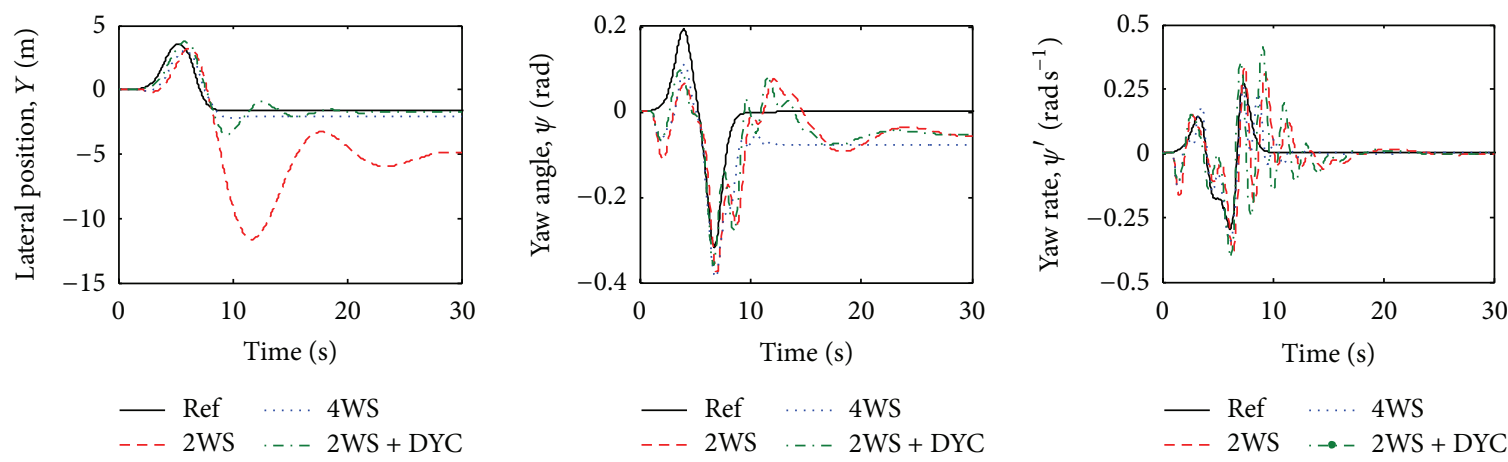

- - 2WS -.. $2 \mathrm{WS}+\mathrm{DYC}$
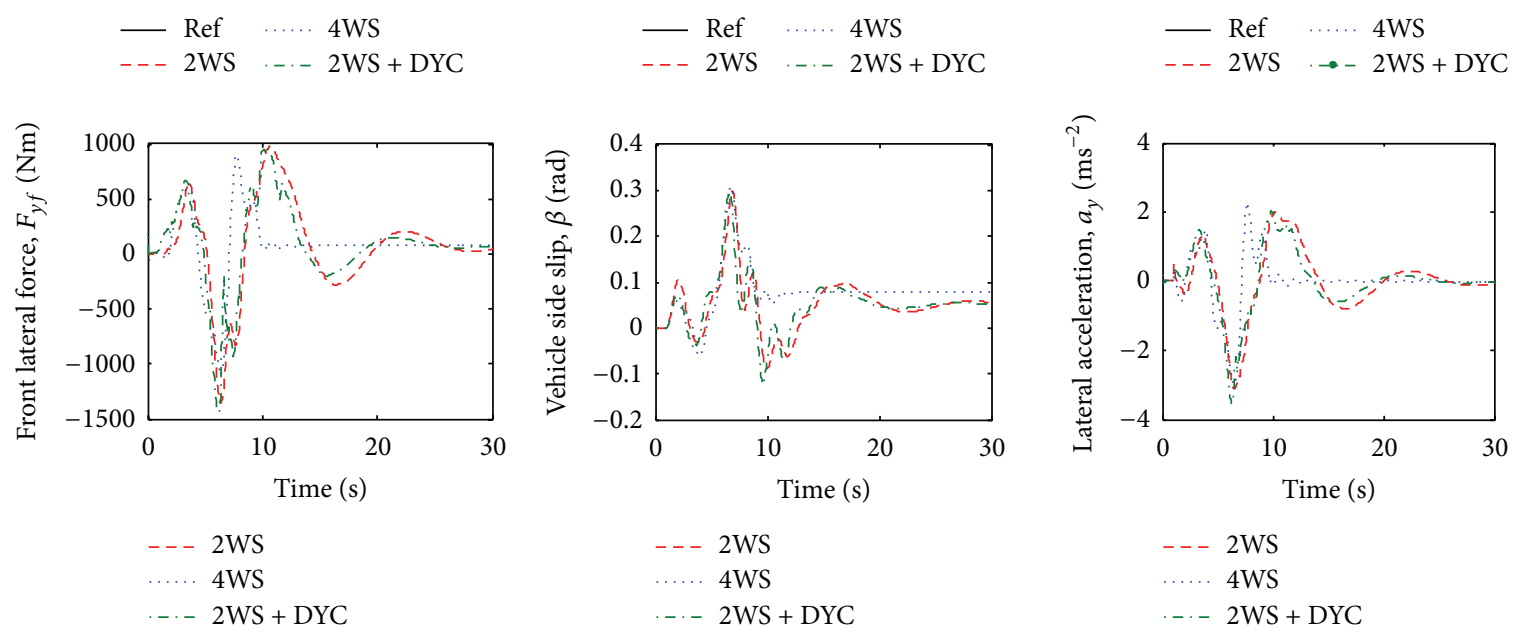

---2 WS

…. $4 \mathrm{WS}$

-. $-2 \mathrm{WS}+\mathrm{DYC}$
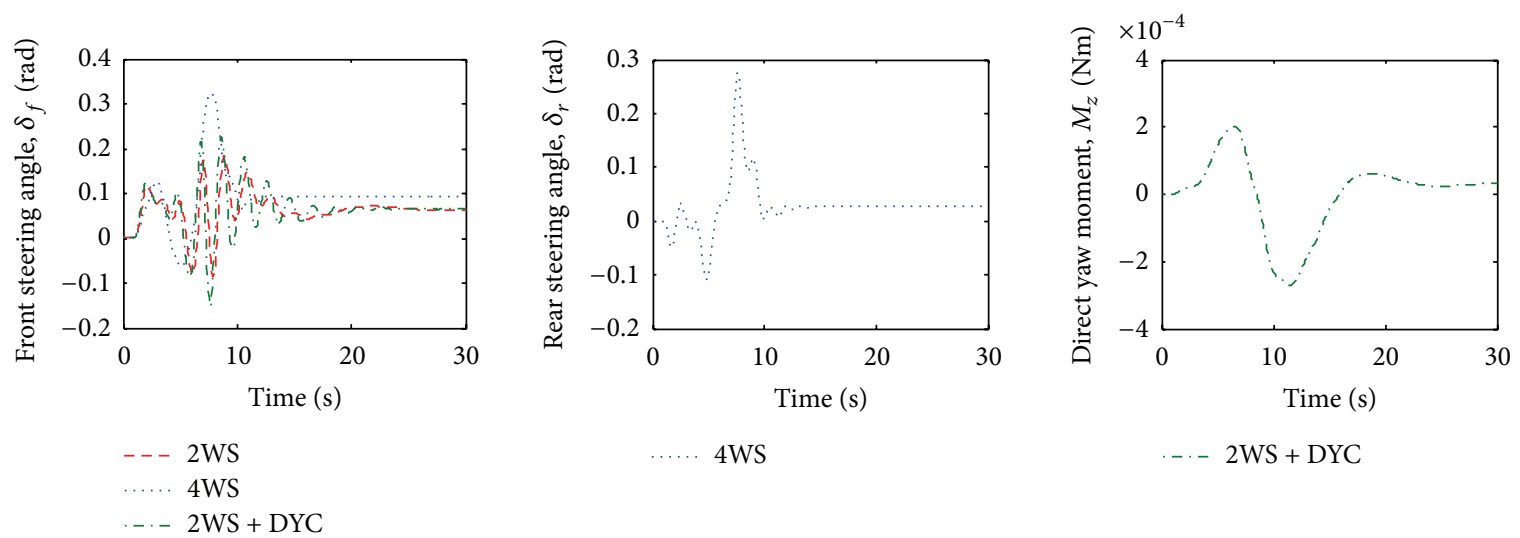

Figure 15: 3DoF controller at $30 \mathrm{~ms}^{-1}$ and $\mu=0.1$ by $2 \mathrm{WS}, 4 \mathrm{WS}$, and $2 \mathrm{WS}+\mathrm{DYC}$.

the car by improving the vehicle handling at high speed, decreasing the turning radius at low speeds, and reducing the tracking error for lateral position and yaw angle response.

The simulation also demonstrated that model predictive control has an ability to reject the side wind effect as a disturbance to the system, which is particularly effective at $10 \mathrm{~ms}^{-1}$ for all cases, except for the 2DoF controller during simulation of higher speeds on lower road surface adhesion. However, both controllers were not able to provide a better response when the vehicle performed at a high forward speed with a low road adhesion coefficient; particularly, when 2DoF controller was simulated, all results showed unstable vehicle response. Thus, for this scenario, we are currently investigating new approaches with a real-time implementable control scheme. Further improvement for future works on control methodology will also be considered to minimize tracking error and to enhance vehicle stability.

\section{Competing Interests}

The authors declare that they have no competing interests.

\section{Acknowledgments}

The authors would like to thank Professor Zidong Wang and Professor Manfred Ploechl for their comments and advice. Special thanks are also extended to Dr. A. Zahran M. Khudzari for his help preparing this paper. 


\section{References}

[1] E. F. Camacho and C. Bordons, Model Predictive Control, Springer, London, UK, 2nd edition, 2007.

[2] C. E. García, D. M. Prett, and M. Morari, "Model predictive control: theory and practice-a survey," Automatica, vol. 25, no. 3 , pp. 335-348, 1989.

[3] P. Mhaskar, "Robust model predictive control design for faulttolerant control of process systems," Industrial \& Engineering Chemistry Research, vol. 45, no. 25, pp. 8565-8574, 2006.

[4] V. Vesely and D. Rosinova, "Robust model predictive control design," in Model Predictive Control, T. Zheng, Ed., pp. 1-24, InTech, Rijeka, Croatia, 2010.

[5] M. Nagai, M. Shino, and F. Gao, "Study on integrated control of active front steer angle and direct yaw moment," JSAE Review, vol. 23, no. 3, pp. 309-315, 2002.

[6] F. Yakub and Y. Mori, "Comparative study of autonomous pathfollowing vehicle control via model predictive control and linear quadratic control," Proceedings of the Institution of Mechanical Engineers, Part D, vol. 229, no. 12, pp. 1695-1714, 2015.

[7] C. March and T. Shim, "Integrated control of suspension and front steering to enhance vehicle handling," Proceedings of the Institution of Mechanical Engineers, Part D: Journal of Automobile Engineering, vol. 221, no. 4, pp. 377-391, 2007.

[8] J. Wang, D. A. Wilson, W. Xu, and D. A. Crolla, "Integrated vehicle ride and steady-state handling control via active suspensions," International Journal of Vehicle Design, vol. 42, no. 3-4, pp. 306-327, 2006.

[9] M. J. L. Boada, B. L. Boada, A. Muñoz, and V. Díaz, "Integrated control of front-wheel steering and front braking forces on the basis of fuzzy logic," Proceedings of the Institution of Mechanical Engineers, Part D: Journal of Automobile Engineering, vol. 220, no. 3, pp. 253-267, 2006.

[10] A. Balluchi, A. Bicchi, and P. Souères, "Path-following with a bounded-curvature vehicle: a hybrid control approach," International Journal of Control, vol. 78, no. 15, pp. 1228-1247, 2005.

[11] K. Moriwaki, "Autonomous steering control for electric vehicles using nonlinear state feedback $H_{\infty}$ control," Nonlinear Analysis, Theory, Methods and Applications, vol. 63, no. 5-7, pp. e2257e2268, 2005.

[12] B. Mashadi, P. Ahmadizadeh, and M. Majidi, "Integrated controller design for path following in autonomous vehicles," SAE Technical Paper 2011-01-1032, SAE International, 2011.

[13] J.-M. Park, D.-W. Kim, Y.-S. Yoon, H. J. Kim, and K.-S. Yi, "Obstacle avoidance of autonomous vehicles based on model predictive control," Proceedings of the Institution of Mechanical Engineers, Part D: Journal of Automobile Engineering, vol. 223, no. 12, pp. 1499-1516, 2009.

[14] Y. Yoon, J. Shin, H. J. Kim, Y. Park, and S. Sastry, "Model-predictive active steering and obstacle avoidance for autonomous ground vehicles," Control Engineering Practice, vol. 17, no. 7, pp. 741-750, 2009.

[15] P. Falcone, F. Borrelli, J. Asgari, H. E. Tseng, and D. Hrovat, "Predictive active steering control for autonomous vehicle systems," IEEE Transactions on Control Systems Technology, vol. 15, no. 3, pp. 566-580, 2007.

[16] T. Keviczky, P. Falcone, F. Borrelli, J. Asgari, and D. Hrovat, "Predictive control approach to autonomous vehicle steering," in Proceedings of the American Control Conference, pp. 46704675, IEEE, Minneapolis, Minn, USA, June 2006.

[17] F. Borrelli, P. Falcone, T. Keviczky, J. Asgari, and D. Hrovat, "MPC-based approach to active steering for autonomous vehicle systems," International Journal of Vehicle Autonomous Systems, vol. 3, no. 2-4, pp. 265-291, 2005.

[18] K. Nam, S. Oh, H. Fujimoto, and Y. Hori, "Robust yaw stability control for electric vehicles based on active front steering control through a steer-by-wire system," International Journal of Automotive Technology, vol. 13, no. 7, pp. 1169-1176, 2012.

[19] H. Yoshida, S. Shinohara, and M. Nagai, "Lane change steering manoeuvre using model predictive control theory," Vehicle System Dynamics, vol. 46, no. 1, pp. 669-681, 2008.

[20] P. Falcone, H. Eric Tseng, F. Borrelli, J. Asgari, and D. Hrovat, "MPC-based yaw and lateral stabilisation via active front steering and braking," Vehicle System Dynamics, vol. 46, no. 1, pp. 611-628, 2008.

[21] W. Kim, D. Kim, K. Yi, and H. J. Kim, "Development of a path-tracking control system based on model predictive control using infrastructure sensors," Vehicle System Dynamics, vol. 50, no. 6, pp. 1001-1023, 2012.

[22] B.-C. Chen and H. Peng, "Differential-braking-based rollover prevention for sport utility vehicles with human-in-the-loop evaluations," Vehicle System Dynamics, vol. 36, no. 4-5, pp. 359389, 2001.

[23] G. Palmieri, P. Falcone, H. E. Tseng, and L. Glielmo, "A preliminary study on the effects of roll dynamics in predictive vehicle stability control," in Proceedings of the 47th IEEE Conference on Decision and Control (CDC '08), pp. 5354-5359, IEEE, Cancun, Mexico, December 2008.

[24] H. Pacejka, Tire and Vehicle Dynamics, Elsevier, Oxford, UK, 3rd edition, 2012.

[25] R. N. Jazar, Vehicle Dynamics Theory and Application, Springer, New York, NY, USA, 3rd edition, 2009.

[26] C. R. Carlson and J. C. Gerdes, "Optimal rollover prevention with steer by wire and differential braking," in Proceedings of the ASME International Mechanical Engineering Congress, vol. 1, no. 1, pp. 345-354, Washington, Wash, USA, November 2003.

[27] C. J. Baker, "Measures to control vehicle movement at exposed sites during windy periods," Journal of Wind Engineering and Industrial Aerodynamics, vol. 25, no. 2, pp. 151-161, 1987.

[28] M. Mahmood and P. Mhaskar, "On constructing constrained control Lyapunov functions for linear systems," IEEE Transactions on Automatic Control, vol. 56, no. 5, pp. 1136-1140, 2011.

[29] M. Mahmood and P. Mhaskar, "Enhanced stability regions for model predictive control of nonlinear process systems," AIChE Journal, vol. 54, no. 6, pp. 1487-1498, 2008.

[30] F. Yakub and Y. Mori, "Heavy vehicle stability and rollover prevention via switching model predictive control," International Journal of Innovative Computing, Information and Control, vol. 11, no. 5, pp. 1751-1764, 2015.

[31] S. Solmaz, M. Corless, and R. Shorten, "A methodology for the design of robust rollover prevention controllers for automotive vehicles with active steering," International Journal of Control, vol. 80, no. 11, pp. 1763-1779, 2007.

[32] D. C. Viano and C. Parenteau, "Case study of vehicle maneuvers leading to rollovers: need for a vehicle test simulating off road excursions, recovery and handling," SAE Technical Paper 200301-0169, SAE International, 2003. 


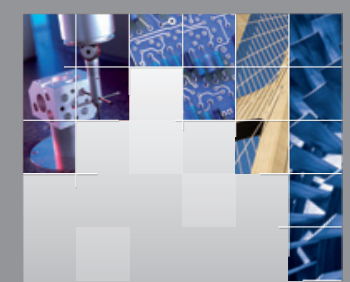

\section{Enfincering}
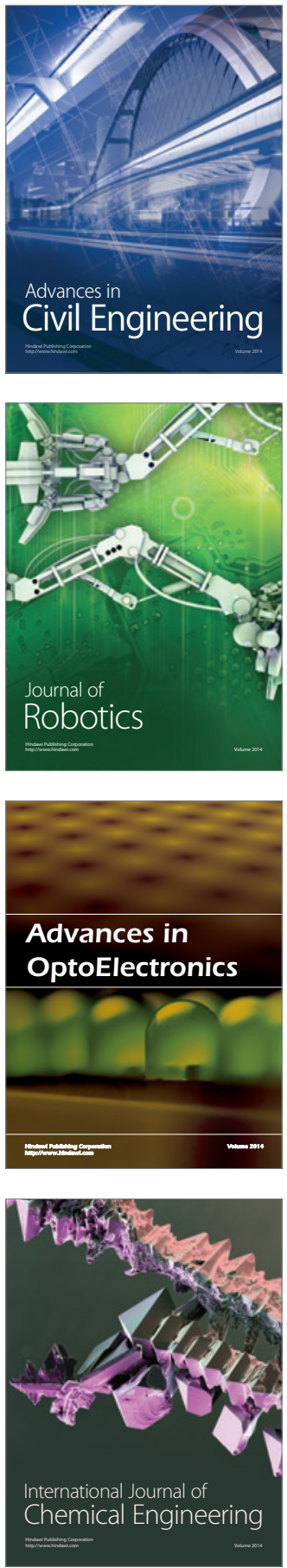

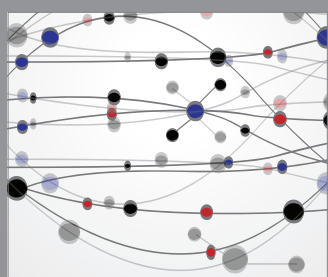

The Scientific World Journal

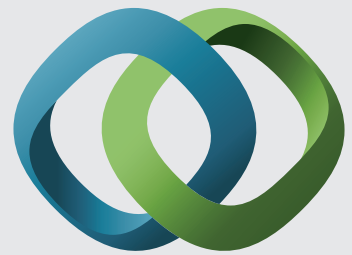

\section{Hindawi}

Submit your manuscripts at

http://www.hindawi.com
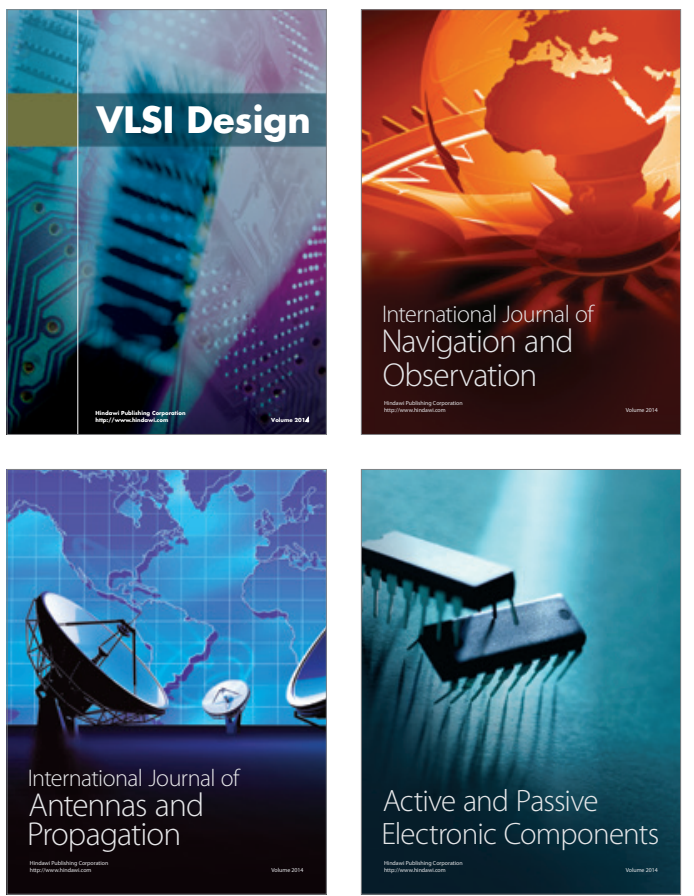
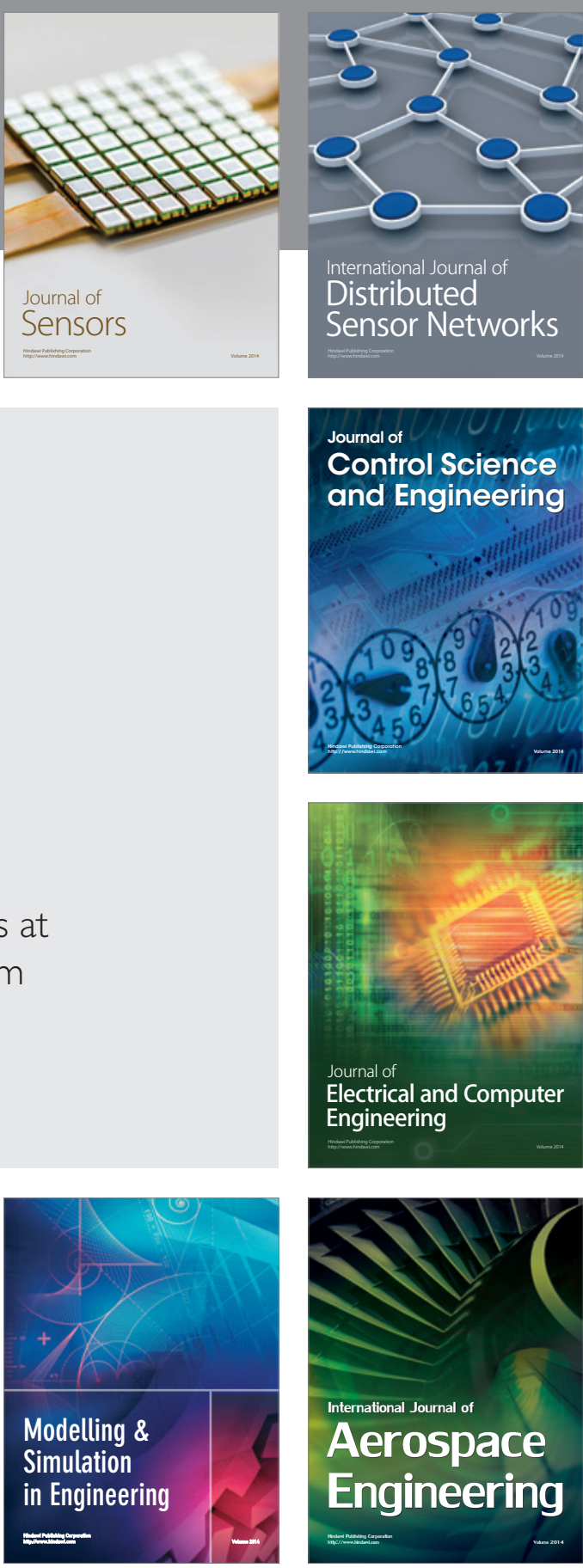

International Journal of

Distributed

Sensor Networks

Journal of

Control Science

and Engineering
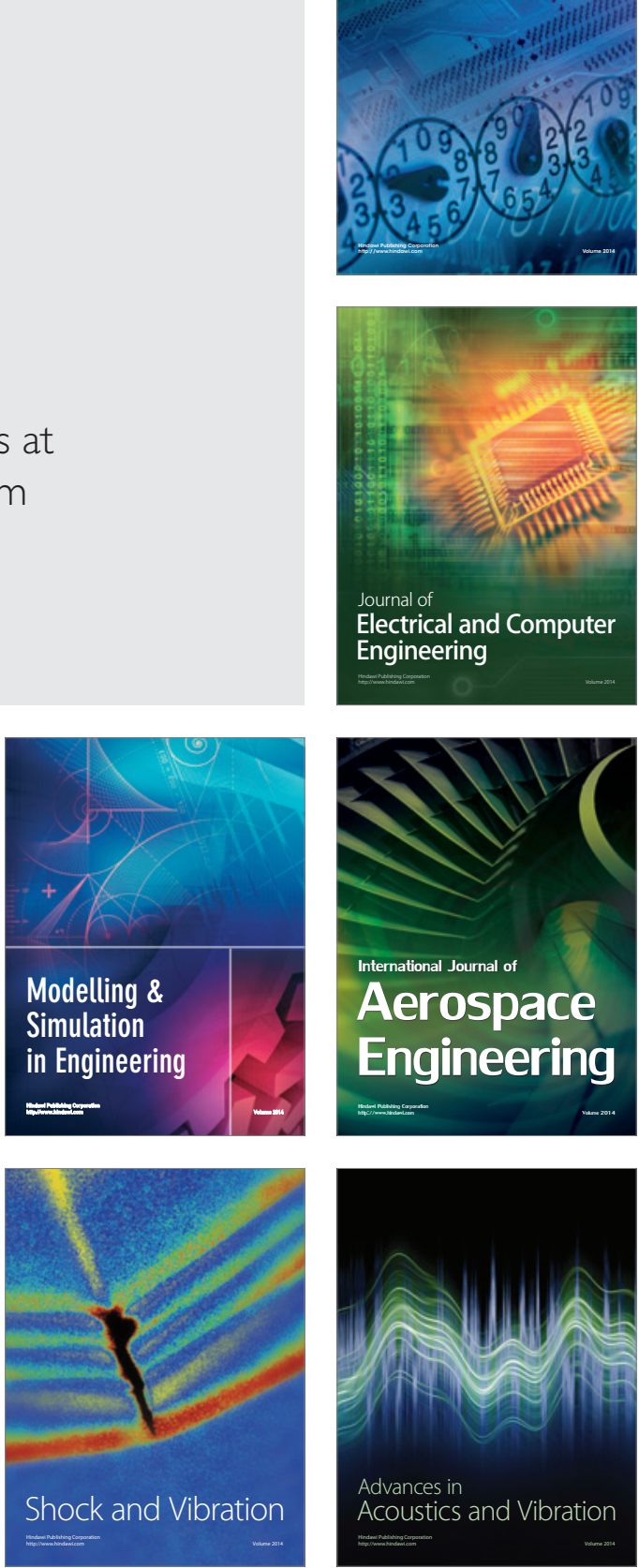